\title{
Dynamic Response of an End-Supported Pontoon Bridge due to Wave Excitation: Numerical Predictions versus Measurements
}

\author{
Knut Andreas Kvåle (i) and Ole Øiseth (iD \\ Norwegian University of Science and Technology, NTNU, Faculty of Engineering, Department of Science and Technology, \\ Trondheim, Norway \\ Correspondence should be addressed to Knut Andreas Kvåle; knut.a.kvale@ntnu.no
}

Received 12 August 2020; Revised 23 February 2021; Accepted 6 May 2021; Published 1 June 2021

Academic Editor: Filippo Ubertini

Copyright (c) 2021 Knut Andreas Kvåle and Ole Øiseth. This is an open access article distributed under the Creative Commons Attribution License, which permits unrestricted use, distribution, and reproduction in any medium, provided the original work is properly cited.

\begin{abstract}
Herein, numerical predictions of the dynamic response of an existing floating pontoon bridge are compared with the measured dynamic response. Hydrodynamic coefficients that describe the fluid-structure interaction and the wave transfer functions are obtained by applying linear potential theory. The results obtained from the hydrodynamic analysis are combined with a beam model of the bridge in a finite element method (FEM) framework to enable stochastic response prediction through the power spectral density method. The standard deviations of the predicted accelerations are compared with the standard deviations of the measured accelerations, and the overall quality of the prediction model is discussed. Predictions with sea states related to the serviceability limit state (SLS) and ultimate limit state (ULS) conditions used in design of the bridge are emphasized. To investigate the behaviour more in depth, a measurement segment is chosen and predictions of the displacement response power spectral density due to excitation characterized by the recorded sea surface elevation are compared with those obtained from the corresponding response measurements. A decent agreement is obtained for both cases when using the model as it is and with waves as the only excitation source, but significant discrepancies are present, in particular, for the torsional components. By including preliminary contributions from wind action and relying on a model optimized against measured modal parameters, a satisfactory agreement is obtained. The effect on the response of an uncertain structural damping is also quantified and concluded to be significant within realistic damping levels.
\end{abstract}

\section{Introduction}

The Norwegian Public Roads Administration (NPRA) is currently conducting a large-scale project to make Coastal Highway E39, which is the highway located along the industry-dense Norwegian west coast, ferry-free. By replacing ferries with permanent road links, the travel time may be reduced by several hours. Many of the straits to cross are deep and wide fjords, which will require an extension of the current bridge technology. Among the possible options are various types of floating bridges, particularly, end-supported solutions with multiple separate pontoons. There are limited numbers of long-span floating road bridges, and such bridges are primarily located in the United States, Canada, Japan, and Norway. Among these bridges, there are two that are end-supported only, namely, the Nordhordland Bridge and the Bergsøysund Bridge, which are both located on the western coast of Norway. Additionally, these two bridges are both based on separate pontoons rather than on a continuous floating pontoon girder. Because these bridges are not supported by side-mooring, they are more flexible and thus susceptible to large displacements and dynamic behaviour. It follows from the above that the available experience regarding the performance of similar structures is very scarce.

Some case studies concerning the response prediction of existing floating bridges are available, e.g., for the Nordhordland Bridge [1], the Bergsøysund Bridge [2-5], the Hood Canal Bridge [6], the William R. Bennett Floating Bridge [7], and the Yumemai Bridge [8, 9]. Recently, studies 
regarding response prediction of planned or generic floating bridges have also emerged in literature, e.g., [10-12]. Based on model tests conducted in an ocean basin as part of NPRAs planning process of the Bergsøysund Bridge, several comparison studies have been conducted $[13,14]$ to provide valuable assessments on the uncertainty involved in the response estimation.

However, few available studies compare the measured response of an existing floating bridge with numerical predictions. The original Hood Canal Bridge in the state of Washington was instrumented with sensors to monitor its response, and these measurements were compared with predictions by [15]. Furthermore, Peterson [16] investigated the performance of mooring cables on the original Evergreen Point Floating Bridge, which is also located in the state of Washington. Thanks to modern sensor technology and a drastic increase in data processing power, it is currently practically possible to handle considerably larger and more accurate sensor networks. The current paper relies on a comprehensive state-of-the-art monitoring system; consequently, this work is able to be more detailed in the comparison of the predicted and measured responses. It can thus provide valuable insight into the expected accuracy of prediction methodology applied for the design of floating bridges.

The accuracy of the prediction methodology is one of the main concerns for ensuring a safe, reliable, and cost-efficient design of new floating bridges. The current paper addresses this concern using a comprehensive model setup [2] of and an extensive monitoring system on the Bergsøysund Bridge [17]. The current paper emphasizes on the accuracy of the prediction methodology by comparison with recorded response, whereas the two referred papers are focused on the modelling methodology and the monitoring system, respectively. For more in-depth details regarding these aspects, the reader is therefore referred to the cited papers. Herein, recorded acceleration quantities are compared to predictions from a numerical model setup in a finite element method (FEM) framework. The bridge is instrumented with sensors that record the response and the excitation, thus making it possible to verify and scrutinize the model and methodology used for the response prediction. Fluid-structure interaction terms and wave excitation transfer functions are estimated by applying linear potential theory. Through the common assumptions of deep water, linear waves, and a stationary and homogeneous wave field, the power spectral density method is applied to predict the response spectral density matrix from a one-point wave spectral density and a directional distribution. Furthermore, by establishing curve fits relating the wave spectrum's peak period and peakedness factor to the significant wave height, the resulting errors on the response are assessed. To address the errors observed in the initial predictions, a more in-depth investigation of the predicted response is carried out on a selected recording. By utilizing an updated model and including approximate wind effects, the discrepancies are reduced. Furthermore, the effect the uncertainty of the structural damping assumed in the model has on the response is quantified by conducting simulations with varying damping levels.
1.1. The Bergsøysund Bridge. The Bergsøysund Bridge is a 933-metre-long end-supported pontoon bridge (Figure 1), with a main span of 830 metres, located on the western coast of Norway. Figure 2 depicts the location of the bridge. The truss superstructure is supported by seven lightweight concrete pontoons. The fact that the bridge is not supported laterally by mooring lines makes it very flexible, and it is, therefore, considered an interesting case study for dynamic analysis. Furthermore, this bridge is the second largest of its kind in existence. The main dimensions of the bridge are depicted in Figure 3.

The excitation and response of the Bergsøysund Bridge are monitored using wave radars, anemometers, accelerometers, and a single global navigation satellite system (GNSS) displacement sensor (Figure 4). For more details regarding the monitoring system, the reader is referred to [17]. Because the wave radar arrangement used in the majority of the recordings is not optimized for characterizing the directional distribution [18], the wave spreading and wave direction are not estimated. The effect that the wave spreading has on the response is not considered to be crucial when the bridge is exposed to wind sea [2]. The monitoring system provides data segmented in 30-minutelong recordings.

\section{Numerical Prediction Model}

The dynamics of floating bridges exposed to first-order wave excitation can be represented using an FEM framework as follows (see, e.g., [19]):

$$
\left[M_{s}\right]\{\ddot{u}(t)\}+\left[C_{s}\right]\{\dot{u}(t)\}+\left[K_{s}\right]\{u(t)\}=\left\{p_{h}(t)\right\},
$$

where $\left[M_{s}\right]$ is the structural mass matrix, $\left[C_{s}\right]$ is the structural damping matrix, $\left[K_{s}\right]$ is the structural stiffness matrix, $t$ is the time variable, $\{u(t)\}$ is the time-dependent displacement vector, $\left\{p_{h}(t)\right\}$ is the time-dependent total hydrodynamic action, and the dot notation is used to denote time differentiation. The total hydrodynamic action $\left\{p_{h}(t)\right\}$, due to a single harmonic wave proportional to $e^{i \omega t}$, can be written as follows (see, e.g., [19]):

$$
\begin{aligned}
\left\{p_{h}(t)\right\}= & \{P(\omega)\} e^{i \omega t}-\left[M_{h}(\omega)\right]\{\ddot{u}(t)\}-\left[C_{h}(\omega)\right]\{\dot{u}(t)\} \\
& -\left[K_{h}\right]\{u(t)\} .
\end{aligned}
$$

Here, $\left[M_{h}(\omega)\right],\left[C_{h}(\omega)\right]$, and $\left[K_{h}\right]$ are the added hydrodynamic mass, hydrodynamic damping, and hydrostatic stiffness, respectively, giving rise to self-exciting forces; $\omega$ is the frequency variable; $i$ is the imaginary unit; and $\{P(\omega)\} e^{i \omega t}$ is the wave excitation due to the monochromatic wave. Through the superposition principle, this is directly interpretable as a general frequency-domain description for irregular stochastic waves. Second-order wave effects and wind excitation are not considered herein because the firstorder wave excitation is assumed to be dominating in the relevant frequency ranges. 


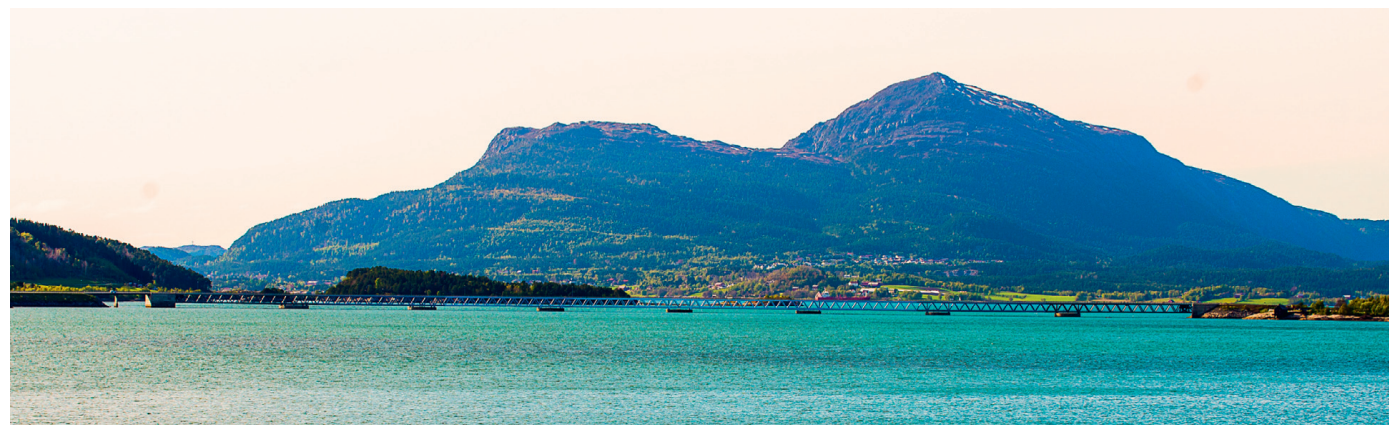

Figure 1: Photograph of the bergsøysund bridge. Photograph by NTNU/K.A. Kvåle.
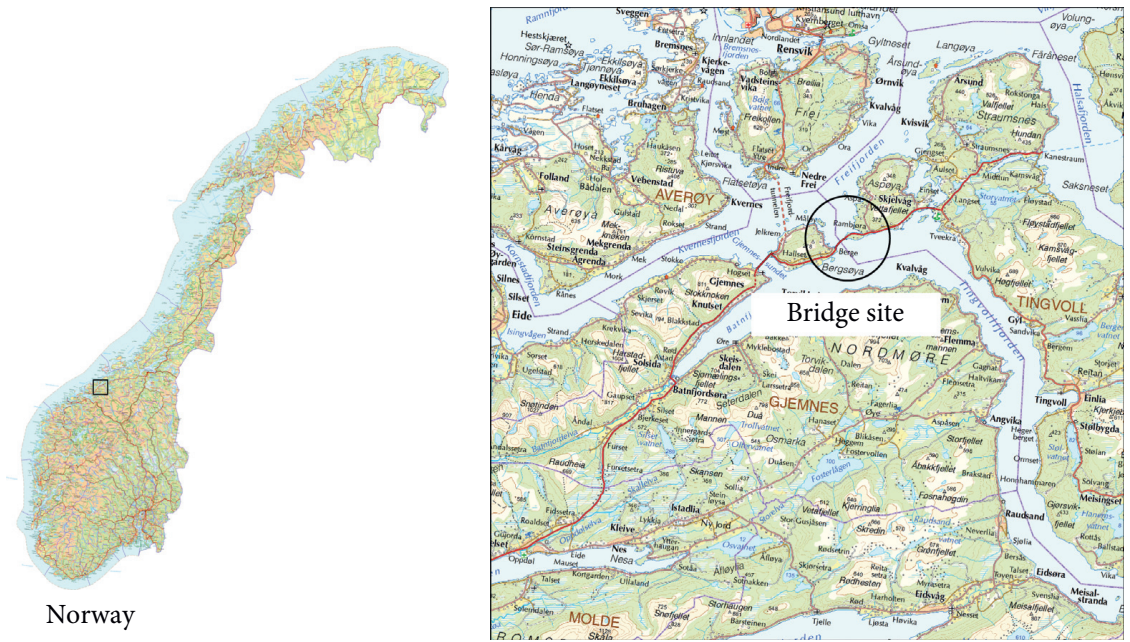

FIgURE 2: Geographic location of the bridge. Map sections: CKartverket (https://www.kartverket.no).

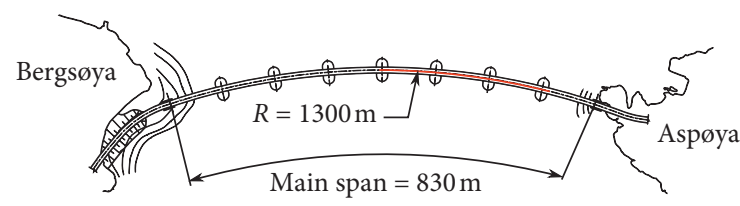

Figure 3: Overhead view of the Bergsøysund Bridge.

2.1. Equation of Motion. By combining equations (1) and (2), the following structure is obtained for the equation of motion:

$$
\begin{aligned}
& \left(\left[M_{s}\right]+\left[M_{h}(\omega)\right]\right)\{\ddot{u}\}+\left(\left[C_{s}\right]+\left[C_{h}(\omega)\right]\right)\{\dot{u}\} \\
& \quad+\left(\left[K_{s}\right]+\left[K_{h}\right]\right)\{u\}=\{P(\omega)\} e^{i \omega t} .
\end{aligned}
$$

To establish the full model, a dry submodel (denoted by subscript $s$ ) and a wet sub-model (denoted by subscript $h$ ), which are described in the two following sections, are combined. For more details about the specifics of this procedure, refer to [2].

2.1.1. Dry Structure: Finite Element Model. An Abaqus FE model consisting of first-order beam elements to represent the truss components and shell elements to represent the deck plate was established. Renderings of the FE model are shown in Figure 5. The stiffness contribution originating from the buoyancy of the pontoons was represented as artificial stiffness matrix contributions to the corresponding nodes in the model. Furthermore, the rigid inertia matrices were included in a similar manner as artificial mass matrix contributions. By conducting an eigenvalue analysis, modal stiffness, modal mass, and eigenvectors were obtained. The corresponding damping contribution was established by assuming a critical damping ratio of $\xi_{0}=0.5 \%$. These results were used to represent the dry part of the combined model setup.

2.1.2. Wet Structure: Hydrodynamic Model. The bridge consists of three different types of pontoons, with varying ballasting, height, and draught. Each of the types are modelled in DNV HydroD, which enables computation of added hydrodynamic mass and damping. The hydrodynamic contributions to all pontoons are thereafter transformed and stacked to correspond to the FE format of the dry structure and finally transformed to the coordinate basis defined by the mode shapes obtained from the eigenvalue solution of the dry submodel, such that the two submodels can be easily combined. This transformation does not represent a true modal decomposition and will therefore not produce diagonal matrices. However, it is a very convenient 


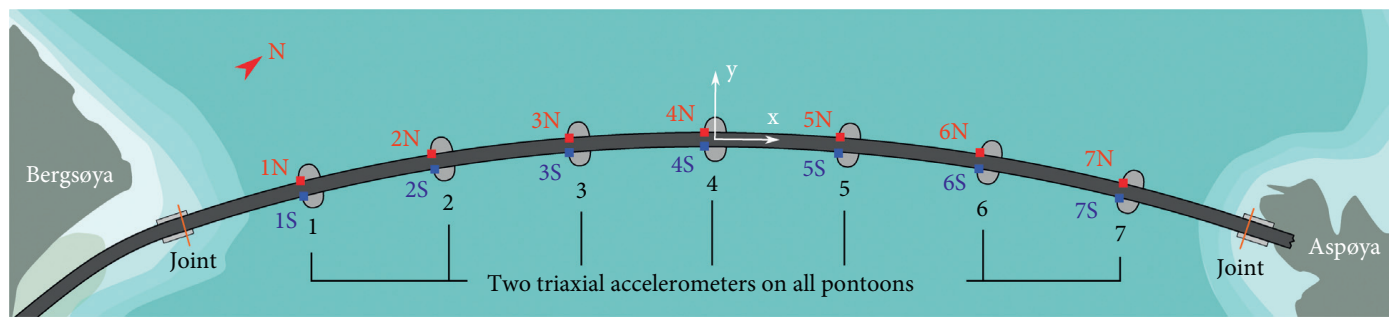

(a)

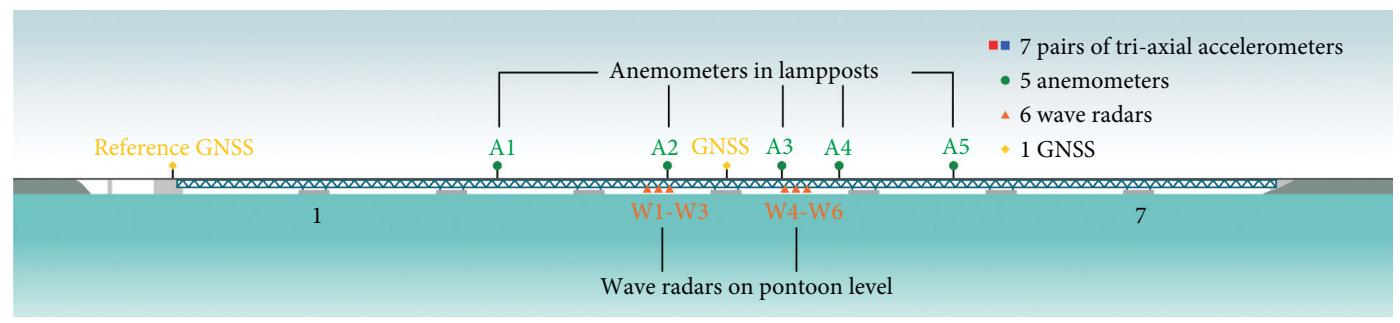

(b)

FIgure 4: Monitoring system operating on the Bergsøysund Bridge which are reproduced from [17] with permission from Elsevier. (a) Top view. (b) Side view.

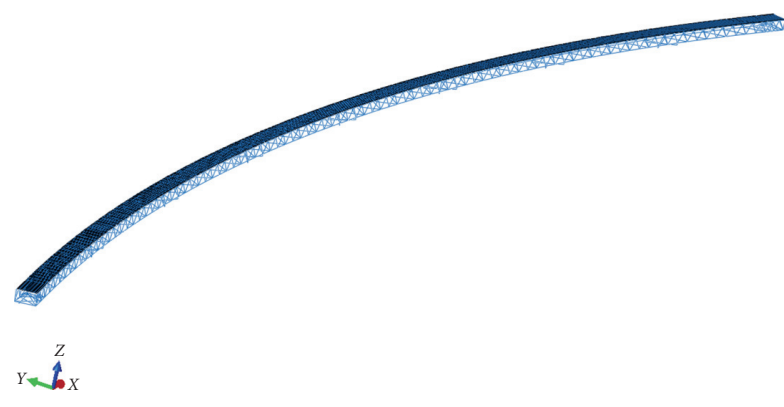

(a)

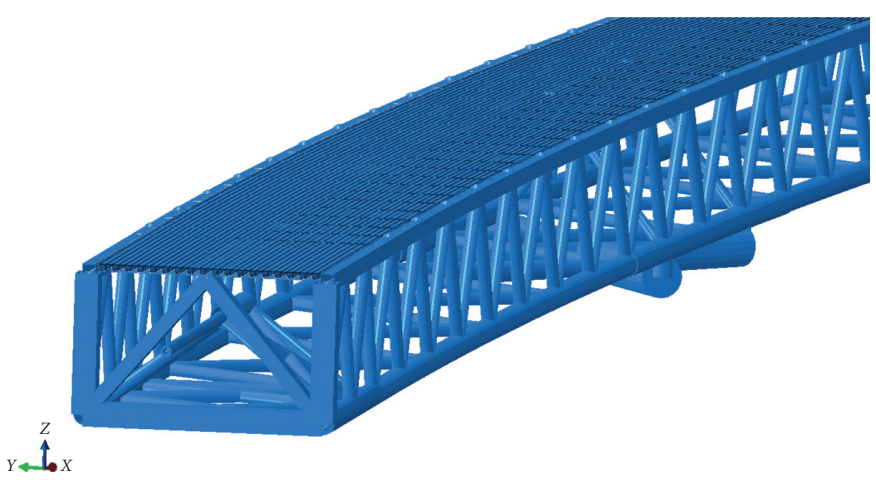

(b)

FIGURE 5: Renderings depicting the finite element model. (a) Truss and deck plate. (b) Close up near end support.

coordinate basis for the purpose of combining the matrices and enabling efficient response predictions.

2.2. Wave Modelling. The cross-spectral density between the wave elevations at points $r$ and $s$ is described as follows (see [1] for more details):

$$
S_{\eta_{r} \eta_{s}}(\omega)=\int_{-\pi}^{\pi} S_{\eta}(\omega) D(\theta) \exp \left(i \frac{|\omega| \omega}{g}\left(\Delta x_{r s} \cos \theta+\Delta y_{r s} \sin \theta\right)\right) \mathrm{d} \theta,
$$

where $S_{\eta}(\omega)$ is the one-point auto-spectral density and $D(\theta)$ is the directional distribution, both equal everywhere under the assumption of homogeneity; $g$ is the gravitational constant; $\Delta x_{r s}=x_{s}-x_{r}, \Delta y_{r s}=y_{s}-y_{r}$; and $\theta$ is the angular variable. In the above equation, it is assumed that the twodimensional wave spectral density is decomposed as $S_{\eta}(\omega, \theta)=S_{\eta}(\omega) D(\theta)$. Furthermore, the cross-spectral densities between the wave excitations on pontoon number $r$ and $s$ (located at points $r$ and $s$ ) are given as follows [1]:

$$
\left[S_{p_{r}, p_{s}}(\omega)\right]=\int_{-\pi}^{\pi}\left\{q_{r}(\omega, \theta)\right\} S_{\eta_{r} \eta_{s}}(\omega, \theta)\left\{q_{s}(\omega, \theta)\right\}^{H} \mathrm{~d} \theta .
$$

Here, $\left\{q_{r}(\omega, \theta)\right\}$ is the hydrodynamic transfer function relating the wave elevation to three forces and three moments acting on pontoon $r, S_{\eta_{r} \eta_{s}}(\omega, \theta)$ is the two-dimensional cross-wave spectral density, and $H$ is the Hermitian operator, which is defined as the complex conjugate and matrix transpose. The hydrodynamic transfer function is established using the numerical hydrodynamic model also used for the self-excited forces. Because there are 7 pontoons in total, there are $7 \times 7=42$ of these matrices to be computed, which are arranged in a global wave excitation spectral density matrix, $\left[S_{p}(\omega)\right]$.

There are two factors in the above equation that affect the final wave excitation: the one-dimensional wave spectral 
density and the directional distribution. Models for these are described in the following two sections.

2.2.1. One-Dimensional Wave Spectral Density. The JONSWAP spectrum is used to define the one-dimensional wave spectral density. The JONSWAP spectrum is defined as follows $[20,21]$ :

$$
\begin{gathered}
S(f)=\alpha g^{2}(2 \pi)^{-4} f^{-5} \exp \left(-\frac{5}{4}\left(\frac{f}{f_{p}}\right)^{-4}\right) \\
\gamma^{\exp \left(-\left(f-f_{p}\right)^{2} / 2 \sigma^{2} f_{p}^{2}\right)} \mathrm{d} \theta,
\end{gathered}
$$

where $f$ is the frequency, $f_{p}$ is the peak frequency, $\gamma$ is the peakedness parameter, $\alpha$ is the Philips parameter, $\sigma=0.07$ for $f<f_{p}$, and $\sigma=0.09$ for $f \geq f_{p}$. This model is highly adjustable, and it is able to describe most monomodal wave spectral densities. However, this ability comes at the cost of the need to determine more parameters, which in principle are co-dependent and vary between excitation situations. The two least intuitively understood parameters are $\gamma$ which characterizes the level of peakedness of the spectrum and $\alpha$ which reflects the fetch characteristics of the wind-wave energy transfer at the site.

2.2.2. Directional Distribution. The $\cos ^{2 s}$ distribution is used to describe the wave spreading and is defined as follows [22]:

$$
D(\theta)=C \cos \left(\frac{\theta-\theta_{0}}{2}\right)^{2 s} .
$$

Here, $C$ is a normalization factor introduced to ensure that $\int_{0}^{2 \pi} D(\theta) \mathrm{d} \theta=1, s$ is the spreading parameter, and $\theta_{0}$ is the mean wave direction. Other distributions might be more appropriate for this application, but the authors believe that the effects caused by other uncontrollable error sources outweigh the effects of possible discrepancies in the model describing the directional distribution. The results in [2] also indicate that the directional distribution does not critically influence the response due to wind wave excitation. A very similar formulation, $D(\theta)=C \cos \left(\theta-\theta_{0}\right)^{n}$, is used in the design basis. Again, $C$ ensures that $\int_{0}^{2 \pi} D(\theta) \mathrm{d} \theta=1$, and $n$ is the spreading parameter. This model is only valid within the angle range $-\pi / 2 \leq \theta-\theta_{0} \leq \pi / 2$ and is assumed zero elsewhere. This model is, therefore, considered to be less elegant than the model in equation (7) and is, therefore, not used for any predictions in this paper.

2.3. Response Prediction. The power spectral density method (see, e.g., [1]) is a highly efficient approach for stochastic response prediction of linear structural systems. This approach is particularly appropriate when the structural system is modelled with frequency-dependent system matrices. The frequency-domain transfer function matrix, $[H(\omega)]$, is easily determined from the total mass, damping, and stiffness matrices. Then, the spectral density of the response is computed as follows:

$$
\left[S_{u}(\omega)\right]=[H(\omega)]\left[S_{p}(\omega)\right][H(\omega)]^{H} .
$$

\section{Sea State}

3.1. Design Basis. According to the design rules used in the design of the Bergsøysund Bridge, a JONSWAP one-dimensional wave spectral density (Equation (6)) and ${\operatorname{a~} \cos ^{n}}^{n}$ directional distribution with sea states specified by the parameters presented in Table 1 should be used. The serviceability limit state (SLS) wave conditions (1-year return period) and the ultimate limit state (ULS) wave conditions (100-year return period) are both given in the table. The provided table specifies ranges for the peak period $T_{p}=1 / f_{p}, \gamma$, and $n$. It is reasonable that an excitation peak period closer to the lowest modes, which, for the current case study, imply large peak periods, is conservative. However, caution should be taken with regard to resonant behaviour; excitation with periods close to periods corresponding to modes of the structure may lead to a large amplification of the response. For the peak periods that provide the largest response, it is reasonable that a more peaked spectral density, represented by a larger $\gamma$, is a conservative choice.

To assist the selection of appropriate parameters for the numerical predictions, a multivariate parameter study was conducted. This is exemplified in Figure 6, which depicts the lateral acceleration standard deviation of pontoon 2, predicted with parameters $\gamma$ and $T_{p}$ varying within the ranges specified in Table 1 . The selected degree of freedom (DOF) revealed the most interesting parameter dependency; a mode with a large contribution in the horizontal response results in a local peak in the response around $T_{p}=3.3 \mathrm{~s}$ (see Figure 7). Note that the lateral component of pontoon 5 exhibits identical behaviour due to the symmetry of the model. All other important DOFs, i.e., the lateral, vertical, and torsional responses of all other pontoons, did not have this pronounced mode-sensitive behaviour. The largest listed peak period for the 1-year sea state $(4.6 \mathrm{~s})$ was, therefore, selected, but the parameter study serves as a reminder of how dynamic problems may behave erratically. $\gamma$ is chosen as the maximum value within the specified range, resulting in the following conservative parameter sets for SLS $\left\{H_{s}=1.00 \mathrm{~m}, \quad T_{p}=4.6 \mathrm{~s}, \quad \gamma=4.5\right\} \quad$ and ULS $\left\{H_{s}=1.41 \mathrm{~m}, T_{p}=5.2 \mathrm{~s}, \gamma=4.5\right\}$. The one-dimensional wave spectral densities corresponding to these two cases are depicted in Figure 8. Note that the above argument is merely based on the standard deviation of the response. In reality, large standard deviation values do not necessarily describe the worst-case situation structurally; the frequency content of the response must also be considered because both the number of cycles in a fatigue analysis and the internal forces are dependent on which vibration modes are excited.

The largest listed spreading parameter is used for all cases, i.e., $n=8$, which in practice is equivalent to a spreading parameter of $s=17$ for the $\cos ^{2 s}$ distribution used in this study. The resulting directional distribution is illustrated in Figure 9. 
TABle 1: Design sea states [23].

\begin{tabular}{lcccc}
\hline Sea state & $H_{s}(\mathrm{~m})$ & $T_{p}(\mathrm{~s})$ & $\gamma$ & $n$ \\
\hline 1-year & 1.00 & $2.9-4.6$ & $2-4.5$ & $2-8$ \\
10-year & 1.23 & $3.1-4.9$ & $2-4.5$ & $2-8$ \\
100-year & 1.41 & $3.3-5.2$ & $2-4.5$ & $2-8$ \\
100-year swell & 0.16 & $6.7-16.0$ & 7 & $10-20$ \\
Abnormal sea state & 1.41 & $5.2-6.7$ & $2-4.5$ & $2-8$ \\
\hline
\end{tabular}

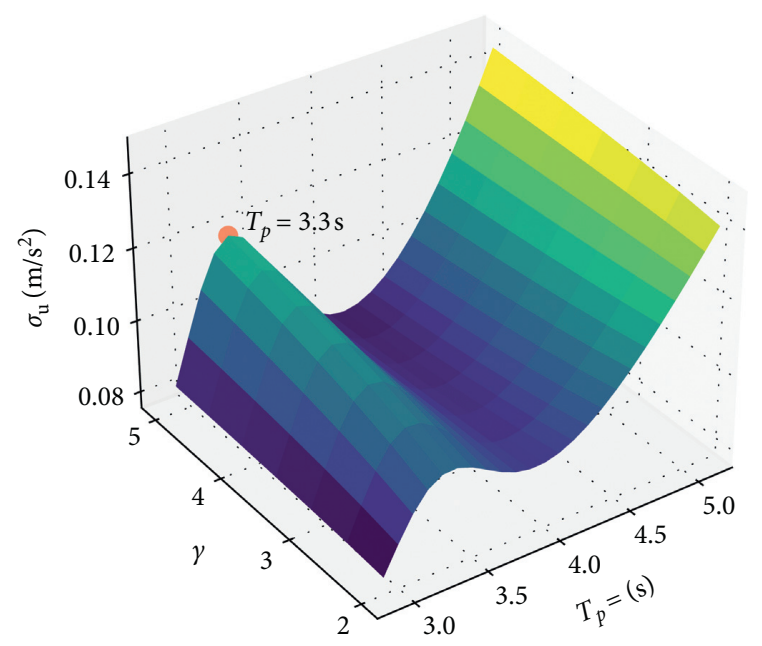

Figure 6: Effect on response standard deviation on lateral acceleration on pontoon 2 due to varying $\gamma$ and $T_{p}$. The spreading parameter $s=17$. The parameters are set according to the ranges specified in Table 1. A local peak is located at approximately $T_{p}=$ $3.3 \mathrm{~s}$ which corresponds to the natural period of mode 10 , as shown in Figure 7.

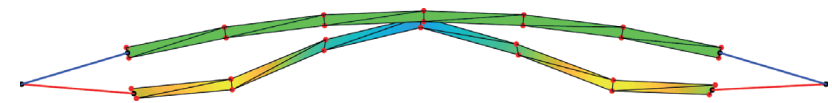

Figure 7: Mode shape of mode 10 (bridge seen from above), with damped natural period, $T_{d}=3.28 \mathrm{~s}$.

3.2. Wave Elevation Measurements. A large amount of recordings including wave elevation measurements was analyzed to estimate the corresponding sea states. Spectral density estimates were established based on wave elevation measurements from all six wave radars for all recordings. For each recording, the average of the spectral densities of all wave measurements was used to fit the JONSWAP spectrum presented in equation (6). Welch's method, with 20 divisions enveloped by Hanning windows and $50 \%$ overlap, was used for all spectral density estimates [24]. Curve fitting was based on minimizing the sum of square errors between the JONSWAP spectrum equation and the spectral density estimated from the wave measurement by applying the Nelder-Mead method, with $\gamma$ and $\alpha$ as the free variables. Curve fits were used rather than the raw spectral density estimates to ensure a smooth spectrum and avoid possible spurious resonant response. The peak period $T_{p}$ was directly determined from the spectral density estimate such that the minimization became more robust. The fitted JONSWAP

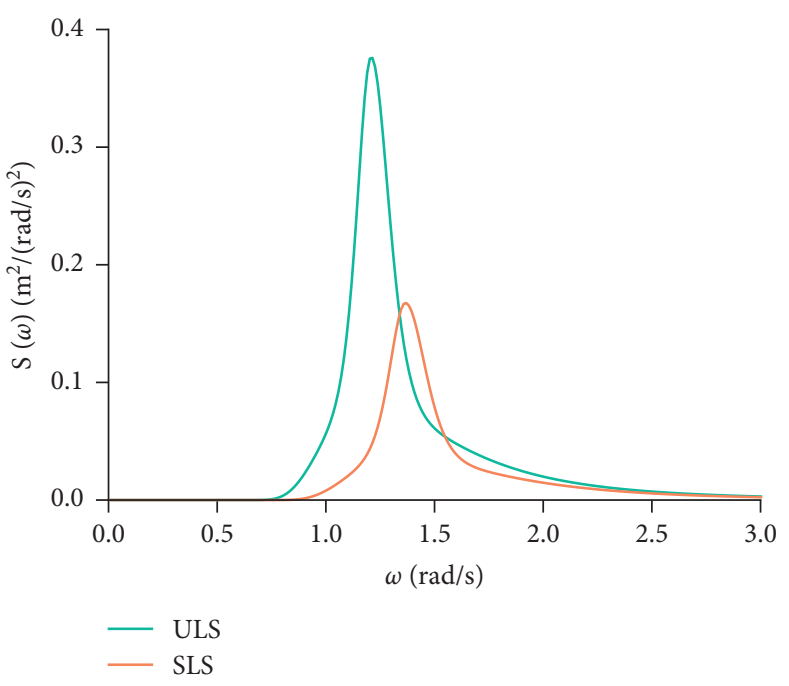

FIgURE 8: JONSWAP spectral densities corresponding to SLS and ULS.

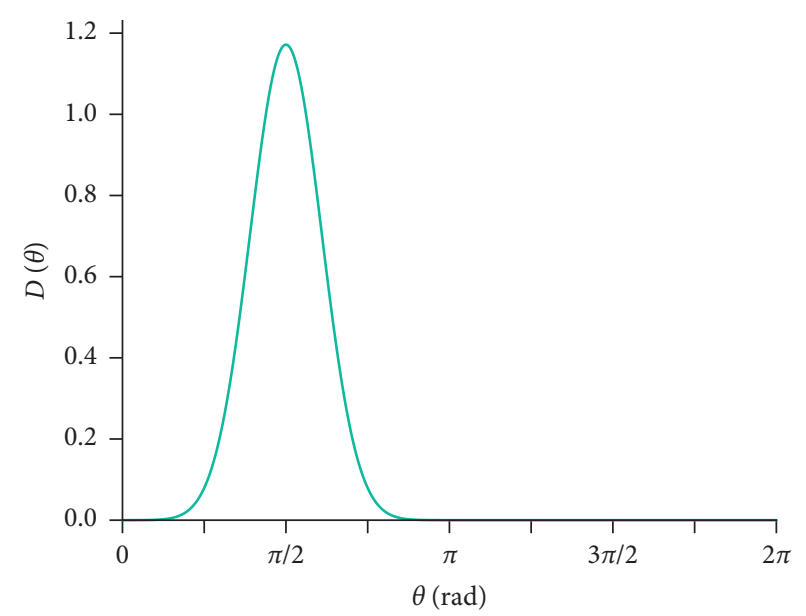

FIgURE 9: $\cos ^{2 s}$ directional distribution with spreading $s=17$ and mean wave direction $\theta_{0}=\pi / 2$.

spectra were thereafter used to characterize the stochastic wave elevations for all the numerical response predictions, which were run for all the recordings. To avoid predictions based on poorly fitted spectral densities to corrupt the results, the goodness of fit was evaluated for every recording. The $R^{2}$-value is used to describe the goodness of fit, and for the present work, the following definition is used [25]:

$$
R^{2}=1-\frac{\sum_{i=1}^{N}\left(f_{i}-y_{i}\right)^{2}}{\sum_{i=1}^{N}\left(y_{i}-\bar{y}\right)^{2}},
$$

where $f_{i}$ is the predicted or fitted value; $y_{i}$ is the measured value, for numerical value $i$ out of $N$ in total; $\bar{y}$ is the average of the measured value; and $R^{2}$ measures the relative amount of variability that can be explained by the prediction or fit. Predictions were only performed when the goodness of fit between the JONSWAP spectrum and the estimated wave spectral density was characterized by $R^{2}>0.8$. 
3.3. Statistical Analysis. The JONSWAP spectra fits of all spectral density estimates of wave elevation were used to study the parameters involved in a statistical manner. $H_{s}$-conditional probability density functions (PDFs) for $T_{p}$ and $\gamma$ were estimated by fitting lognormal PDFs to the histograms of the two parameters for specified ranges of $H_{s}$. The results are shown in Figure 10. The data points for $H_{s}$ and $T_{p}$ are overlaid on the plot in Figure 10(a) to indicate the appearance of the raw data.

The effect $\gamma$ has on the spectrum is illustrated in Figure 11 , with $H_{s}=0.68 \mathrm{~m}$ and $T_{p}=2.54 \mathrm{~s}$. To justify the use of a lognormal distribution, a lognormal distribution is fitted to a scaled histogram of wave measurements satisfying $\gamma<6$ for all significant wave height values. The result is depicted in Figure 12 and indicates that the chosen lognormal distribution is appropriate. A similarly good fit is obtained for $T_{p}$. By performing linear curve fits to the ridges of the PDFs in Figure 10, functions that relate the two parameters to the significant wave height were established. This enabled the wave spectral density to be fully described by the significant wave height. It is noted that the specific fitted functions are only useful for this particular site, but similar procedures could be useful in exploratory stages of design of other structures as well. The value of $\alpha$ in the JONSWAP spectrum is computed directly from the values of $H_{s}$ and $\gamma$ [21].

\section{Dynamic Response}

In the following section, predicted and measured response values are compared. First, a comparison in terms of standard deviations of the bulk of the recordings is made. Second, the spectral densities estimated from a selected 30min recording are compared with the measured spectral densities. Note that all response values are presented relative to a local coordinate system of each pontoon, where the $x$ axis is specified as along the tangent of the bridge main axis.

4.1. Standard Deviations of All Recordings. As discussed in Section 3.2, JONSWAP spectra were fitted to measured wave elevations for all recordings. The JONSWAP spectra were thereafter used as input in response predictions following the procedure outlined in Section 2, to produce relevant response estimates. The significant wave height, $H_{s}$, is, for the current case study, the most important input parameter in the wave excitation model, and it is assumed to be the controlling parameter for the global statistical assessment of the response prediction. The relation between $H_{s}$ and the resulting response can be illustrated in scatter plots, to indicate the relationship. Because $H_{s}$ does not sufficiently describe the sea state by itself, it is reasonable that variability in the measured response is observed for a given value of $H_{s}$. The peakedness of the wave spectral density, the directional spreading and mean angle of the waves, and the inhomogeneity in the wave field will all contribute to the variability observed in the measured response. Furthermore, other excitation sources, such as wind and traffic, will produce excitation that is unaccounted for and that in turn is observed as variability.
It can be shown that the response of the bridge predicted using a first-order model setup is linearly dependent on the significant wave height, given that the shape of the wave spectral density otherwise remains the same. However, the shape and peak period of the wave spectral density are normally dependent on the significant wave height, such that, in sum, a nonlinear trend is expected.

In addition to all established sea state parameters obtained from the measurements, the following three different parameter sets were used as input for numerical predictions of the dynamic response:

(a) SLS design parameters, 1-year sea state, $T_{p}=4.6 \mathrm{~s}$, $\gamma=4.5$, and $H_{s}=1.0 \mathrm{~m}$

(b) ULS design parameters, 100-year sea state, $T_{p}=5.2$ s, $\gamma=4.5$, and $H_{s}=1.41 \mathrm{~m}$

(c) Parameters as functions of $H_{s}$, as identified in Section 3.3 and depicted in Figure 10, $T_{p}=1.53+$ $1.93 H_{s}$ and $\gamma=1.32+0.61 H_{s}$

All predictions were based on a directional distribution characterized by spreading parameter $s=17$ and head sea $\left(\theta_{0}=90^{\circ}\right)$, as depicted in Figure 9.

Figures 13-15 show the resulting predicted standard deviations of the lateral, vertical, and torsional accelerations, respectively, of pontoons 2-4 compared to the corresponding measured accelerations. As the response of the bridge is mainly monitored by accelerometers, comparing the accelerations is considered better than comparing the displacements. Displacements would have to be estimated by numerical integration of accelerations; the well-known issues of drift and inaccuracies of low-frequency components would potentially introduce large errors in the standard deviations. Establishing the acceleration response from the numerical prediction is, on the other hand, straightforward. The figures indicate a very good agreement between measured and predicted lateral acceleration response. The predicted and measured vertical acceleration are also in decent agreement. The torsional acceleration response, however, appear significantly underpredicted compared to its measured counterpart.

The figures also compare the measured responses with the response predicted using the three JONSWAP parameter sets denoted as $a-c$. The response prediction based on parameters of the SLS sea state $(a)$, with $H_{s}=1.0 \mathrm{~m}$, agrees well with the response for this excitation level based on both the fitted parameters and the full parameter set (blue dots) for most DOFs. The quality of the ULS $(b)$ prediction is more difficult to assess because the specified excitation level, i.e., $H_{s}=1.41 \mathrm{~m}$, is far above what is observed for any of the recordings. The plots reveal that the predictions based on the fitted $\gamma$ and $T_{p}$ values (c) generally produce response values that are very close to those from the predictions based on using all parameters. As mentioned above, the torsional response is underpredicted. Because the predicted torsional response based on the parameter fits matches the predictions from the full parameter set very well, the underestimation error is likely due to model inaccuracies (excitation model or structural model) rather than inaccuracies in the description 


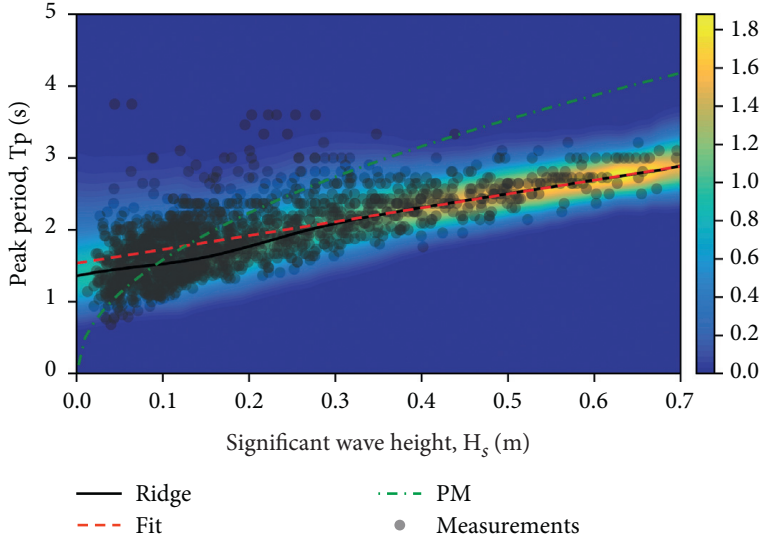

(a)

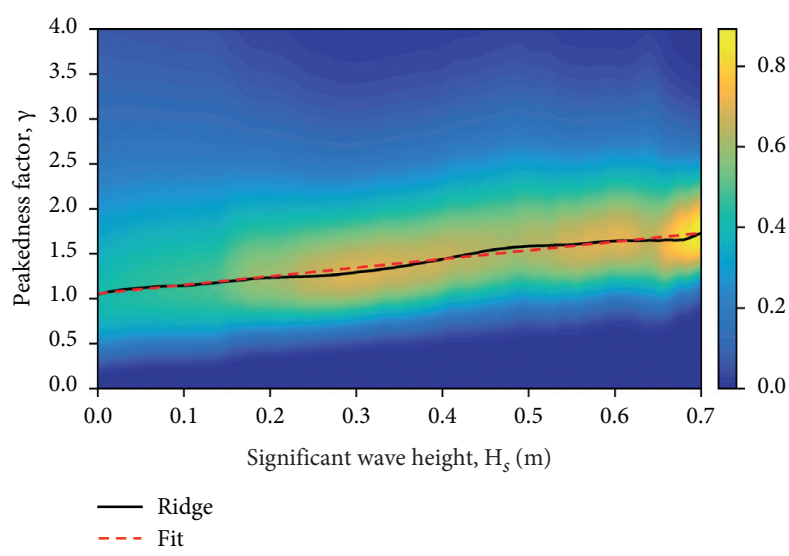

(b)

Figure 10: Estimated $H_{s}$-conditional probability density functions for peak period and peakedness factor. Both plots are based on fitting a lognormal distribution to the data points The data used are restricted to having a good fit to the JONSWAP spectrum, i.e., with $R^{2}>0.8$. Furthermore, a linear fit to the ridge of the probability density function is computed and shown in the plots. This fit is based on the subdomain of the probability density function, where $H_{s}>0.4 \mathrm{~m}$. (a) $f_{T_{p} \mid H_{s}}\left(t_{p}, h_{s}\right)$. The fitted equation reads out $T_{p}=1.53+1.93 H_{s}$. The Pierson-Moskowitz (denoted PM) relation between peak period and significant wave height is also given. The data points are indicated by dots. (b) $f_{\gamma \mid H_{s}}\left(\gamma, h_{s}\right)$. The fitted equation reads out $\gamma=1.32+0.61 H_{s}$.

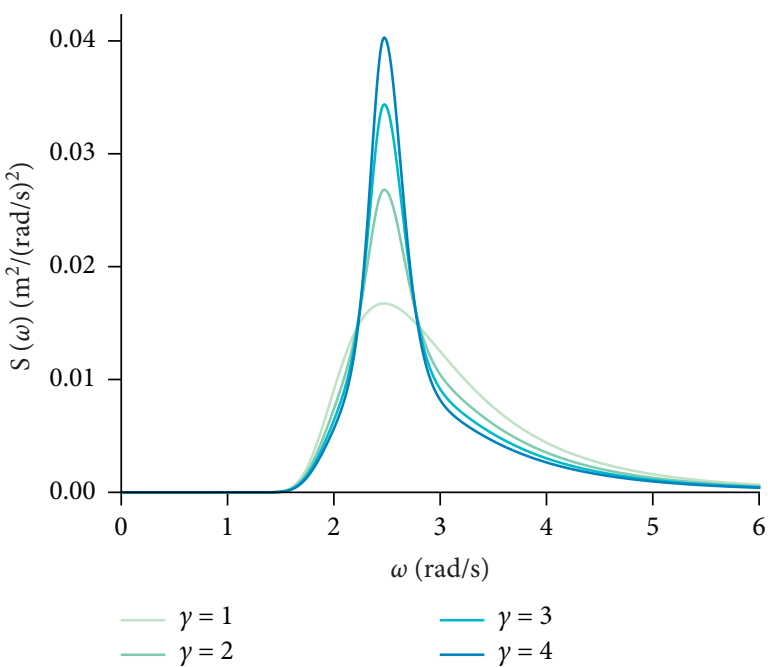

FIGURE 11: JONSWAP spectrum for varying $\gamma$ values. $H_{s}$ and $T_{p}$ are set equal to $0.68 \mathrm{~m}$ and $2.54 \mathrm{~s}$, respectively.

of the sea state. The general variability observed in the predictions based on the full parameter set is, therefore, assumed to be highly influenced by the variability in the two parameters $\gamma$ and $T_{p}$. Note that error sources explaining the discrepancy between the predictions and the measurements, such as model errors, possible nonlinearities, other excitation sources, errors in the directional distribution, and inhomogeneities, will not affect the goodness of fit between the responses computed using the simplified and full parameter sets.

The $R^{2}$ value between the measured and predicted responses is computed for all measured DOFs and is presented in Figure 16. Because many recordings exist in the low-excitation

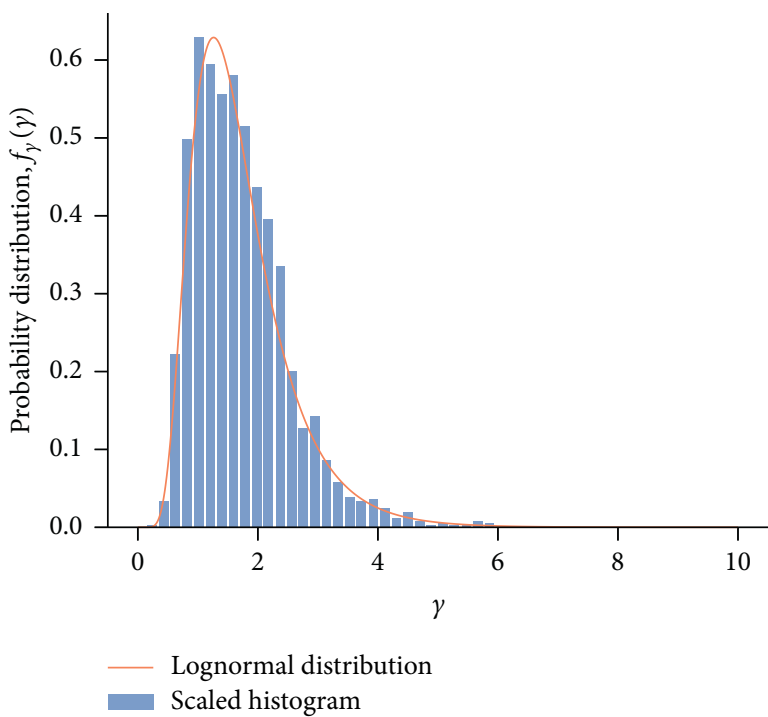

Figure 12: Fit of $\gamma$ from the JONSWAP spectra to a lognormal distribution. The set of obtained JONSWAP parameters used for the fitting were restricted to $\gamma<6$ and $R^{2}>0.8$.

regime, which would heavily influence the results, only recordings with $H_{s}>0.15 \mathrm{~m}$ is included in the computation of $R^{2}$. This figure can be used to draw the same conclusion as from the other comparisons: the lateral acceleration ( $y$ in figure) is fairly well predicted, the vertical acceleration ( $z$ in figure) is decently predicted, and the torsional acceleration $\left(\theta_{x}\right.$ in figure) is predicted with an accuracy that is below satisfactory. Furthermore, the figure reveals that the goodness of fit of the most important acceleration components, namely, the lateral, vertical, and torsional accelerations, are close to symmetrical. It thus implies that there are no systematic asymmetric inhomogeneities across the strait, as the predictions are based on 


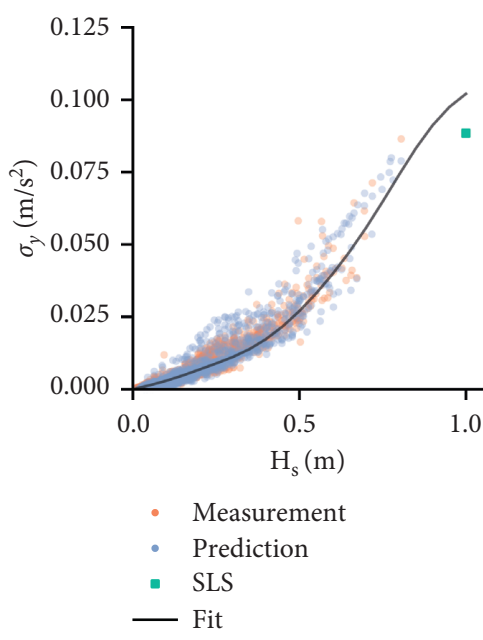

(a)

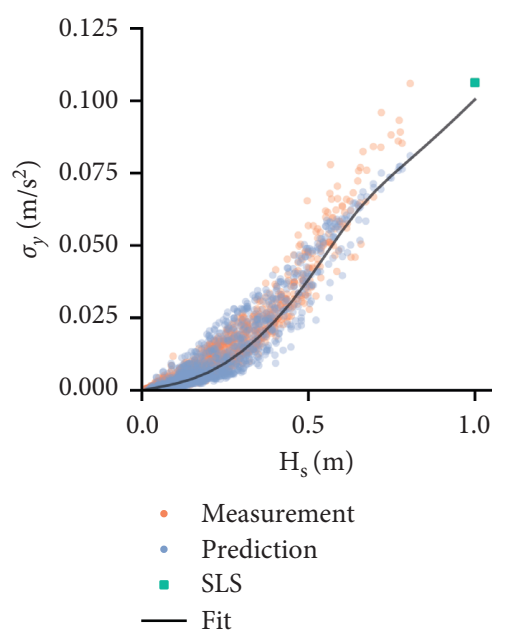

(b)

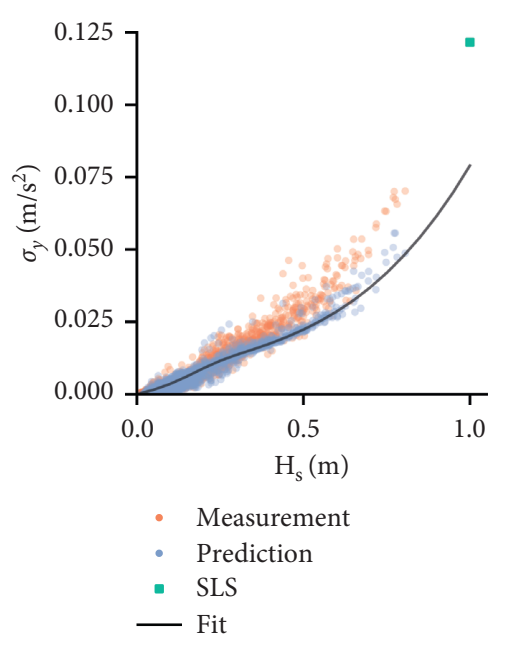

(c)

FIGURE 13: Comparison between the measured and predicted standard deviations of lateral accelerations. The points corresponding to the ULS $\left(H_{s}=1.41 \mathrm{~m}\right)$ are located outside the limits of the plots and are therefore indicated in the caption (see parentheses). (a) Pontoon 2 $\left(0.204 \mathrm{~m} / \mathrm{s}^{2}\right)$. (b) Pontoon $3\left(0.307 \mathrm{~m} / \mathrm{s}^{2}\right)$. (c) Pontoon $4\left(0.405 \mathrm{~m} / \mathrm{s}^{2}\right)$.

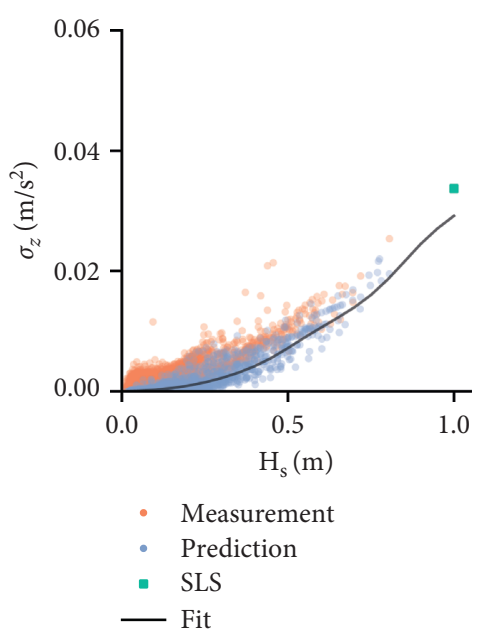

(a)

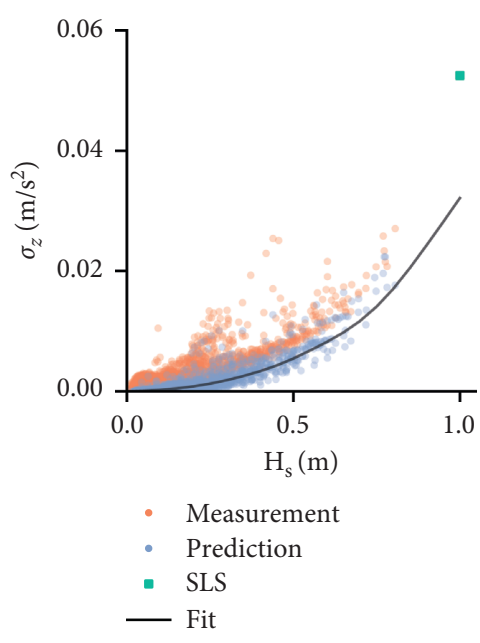

(b)

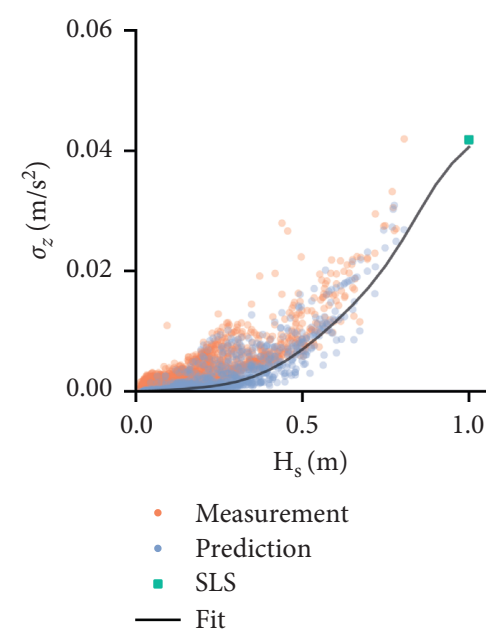

(c)

FIgURE 14: Comparison between the measured and predicted standard deviations of vertical accelerations. The points corresponding to the ULS $\left(H_{s}=1.41 \mathrm{~m}\right)$ are located outside the limits of the plots and are as follows. (a) Pontoon $2\left(0.143 \mathrm{~m} / \mathrm{s}^{2}\right)$. (b) Pontoon $3\left(0.120 \mathrm{~m} / \mathrm{s}^{2}\right)$. (c) Pontoon $4\left(0.156 \mathrm{~m} / \mathrm{s}^{2}\right)$.

fully homogeneous conditions. If systematic inhomogeneities exist, they are, therefore, likely to be fairly symmetric. It is also observed that the $R^{2}$ value that corresponds to the longitudinal acceleration of the fourth pontoon is negative, which is a consequence of how $R^{2}$ is defined in equation (9): negative values simply imply that the average of the measurements is a better fit to the data than the prediction.

4.2. Selected Recording. A recording initiated on December 30, 2015, $18: 25$ (local time), was chosen for further study. Fundamental statistics from the recording are presented in Table 2.
To justify selecting a spreading parameter value $s$ without supporting the choice by data, the effect of changing spreading parameter on the acceleration response is illustrated in Figure 17. This figure is consistent with the findings in [2] of a low sensitivity to changing spreading parameter of response driven by locally generated wind waves. The mean wave direction is assumed lateral, i.e., $\theta_{0}=90^{\circ}$, which is considered to be a conservative choice. Cancellation effects on symmetric modes due to the perfect head sea are not considered significant for short-crested sea states, as considered herein. Furthermore, due to the geography surrounding the bridge, it is unlikely that large waves are approaching the bridge with very oblique angles. However, isolated, 


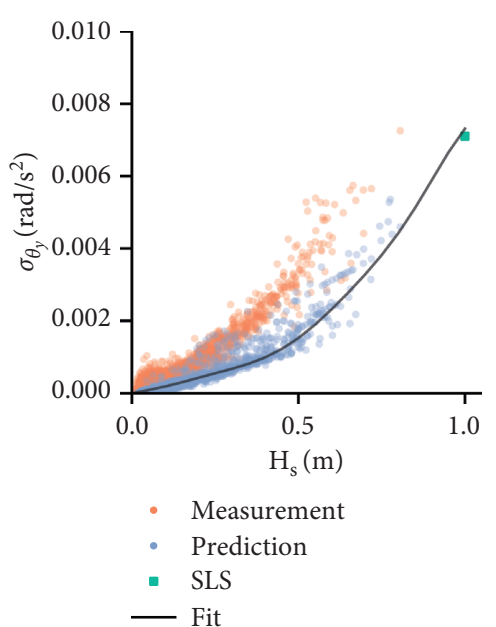

(a)

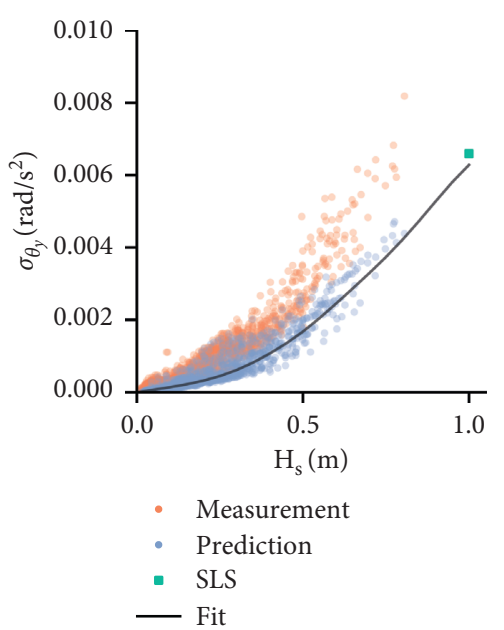

(b)

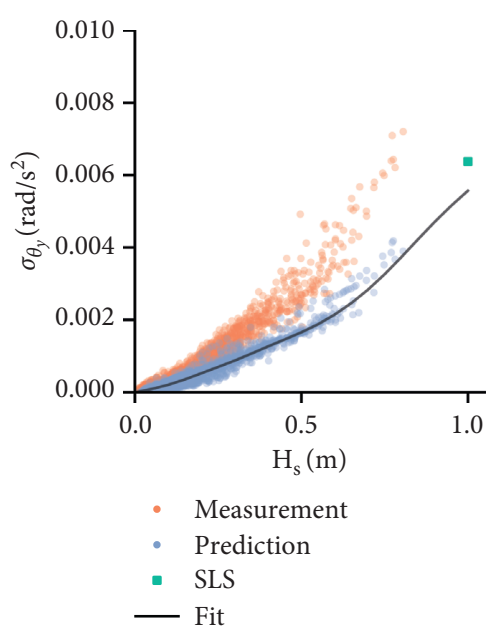

(c)

FIGURE 15: Comparison between the measured and predicted standard deviations of torsional accelerations. The points corresponding to the ULS $\left(H_{s}=1.41 \mathrm{~m}\right)$ are located outside the limits of the plots and are as follows. (a) Pontoon $2\left(0.020 \mathrm{rad} / \mathrm{s}^{2}\right)$. (b) Pontoon $3\left(0.021 \mathrm{rad} / \mathrm{s}^{2}\right)$. (c) Pontoon $4\left(0.021 \mathrm{rad} / \mathrm{s}^{2}\right)$.
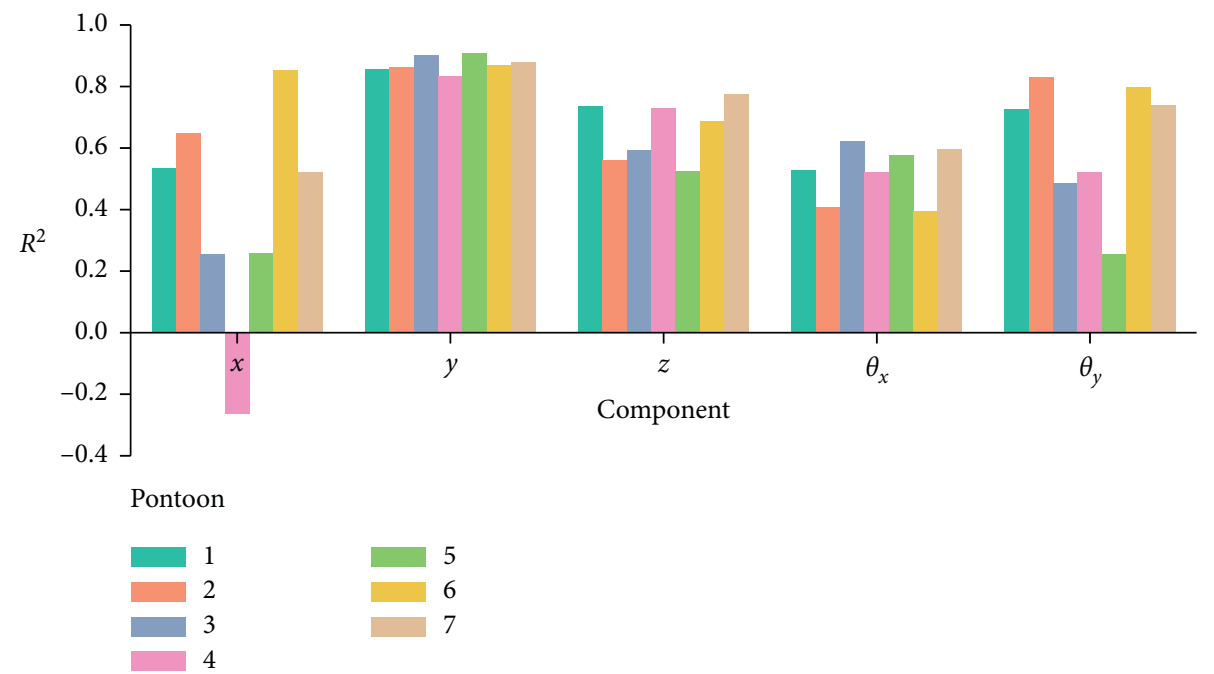

FIGURE 16: Coefficient of determination between the standard deviations of the measured accelerations and the corresponding predictions based on a JONSWAP fit. Only recordings with $H_{s}>0.15 \mathrm{~m}$ are retained to avoid the large cluster of low-excitation recordings from corrupting the result. The components are indicated under the bars corresponding to the first pontoon.

asymmetric modes could respond more from waves with oblique mean angles.

The average of the spectral densities estimated from wave elevation of all wave radars were used to estimate parameters of the JONSWAP spectrum. Both are depicted in Figure 18.

Figures 19-21 show the spectral densities of predicted and measured lateral, vertical, and torsional displacement components of pontoons 2-4. A sensitivity-based model update was performed on the same model in [4], and the response predictions using the updated model are also given in the figures together with the results from the original model. The original model is still considered relevant in the comparison, as this represents the accuracy that can be expected when establishing numerical models for design purposes. Note that the $y$-axis in the three figures have different scalings. To establish displacement estimates of the measured acceleration, the acceleration spectral densities are multiplied by a factor of $\omega^{-4}$. This process introduces errors, particularly, in the low-frequency domain; the frequency range below $0.5 \mathrm{rad} / \mathrm{s}$ of the spectral densities is, therefore, not given much attention. It is worth noting that the response in the mentioned region does not contribute considerably to the acceleration standard deviations compared in the preceding section.

The figures indicate a relatively poor agreement between measurements and the predictions based on the original model, particularly, for vertical and torsional response; the natural frequencies of several modes are obviously not 
TAвLE 2: Fundamental statistics from the selected recording. Listed acceleration and displacement values refer to standard deviations. Acceleration and displacement values describe the local motion of pontoon 3 . Wind direction is defined as clockwise increasing with $0^{\circ}$ along the tangent at midspan.

\begin{tabular}{lcccccccc}
\hline & \multicolumn{2}{c}{ Wave height $(\mathrm{cm})$} & \multicolumn{2}{c}{ Wind } & \multicolumn{2}{c}{ Acceleration $(\mathrm{mg})$} & \multicolumn{2}{c}{ Displacement $(\mathrm{mm})$} \\
Time segment & Significant & Maximum & Speed $(\mathrm{m} / \mathrm{s})$ & Direction [ ${ }^{\circ}$ ] & Lateral & Vertical & Lateral & Vertical \\
\hline 0-10 minutes & 59.2 & 105.4 & 16.6 & 104.1 & 3.9 & 1.4 & 7.9 & 6.5 \\
10-20 minutes & 70.2 & 129.8 & 17.8 & 104.2 & 6.2 & 1.5 & 12.8 & 5.7 \\
20-30 minutes & 72.6 & 130.3 & 17.3 & 105.1 & 8.5 & 1.7 & 16.7 & 6.9 \\
\hline
\end{tabular}

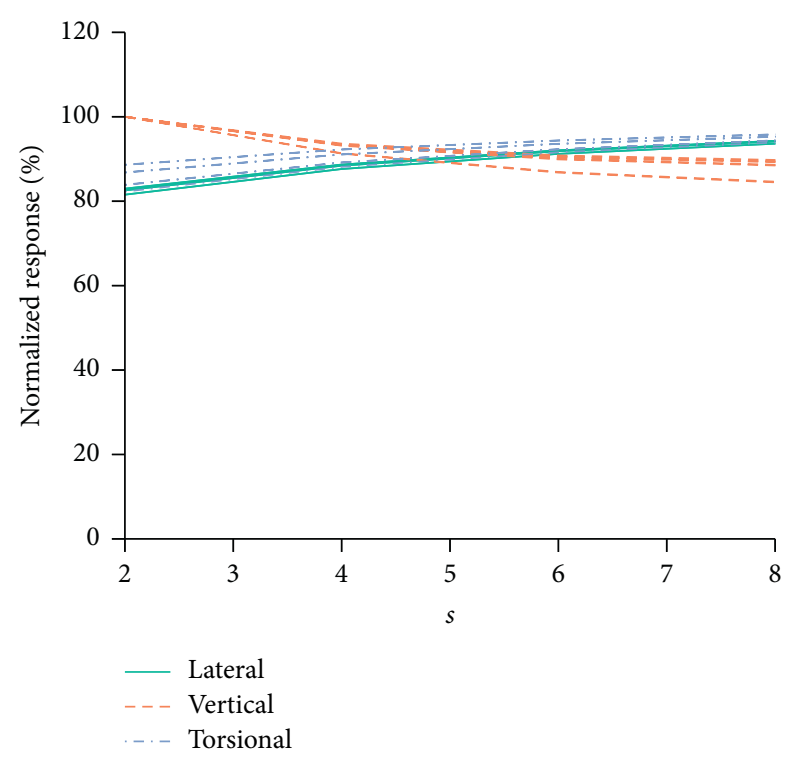

FIGURE 17: Effect of spreading parameter on the standard deviations of lateral, vertical, and torsional accelerations. The standard deviations are normalized such that they are unity for the spreading parameter value of which they are at their maximum.

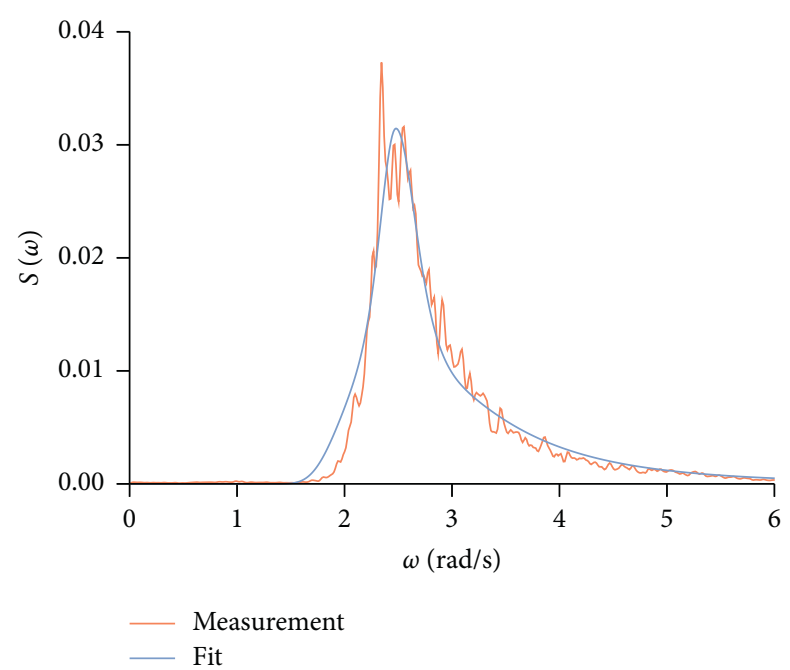

FIGURE 18: Fitting of JONSWAP spectrum to the average of the power spectral density estimates obtained from the wave radars. The fitted curve corresponds to the values $H_{s}=0.68 \mathrm{~m}, \gamma=2.55$, and $T_{p}=2.54 \mathrm{~s}$. The spectral estimates are obtained by applying Welch's method with 20 divisions, Hanning window, and $50 \%$ overlap.

consistent with the measurements. As seen in the figures, the update of the model drastically improves the result, in general ensuring a good agreement for all three investigated components in frequency regions above $1.5 \mathrm{rad} / \mathrm{s}$. The critical damping ratio applied to the dry part of the structure, $\xi_{0}=0.5 \%$, is considered highly uncertain. As the most 


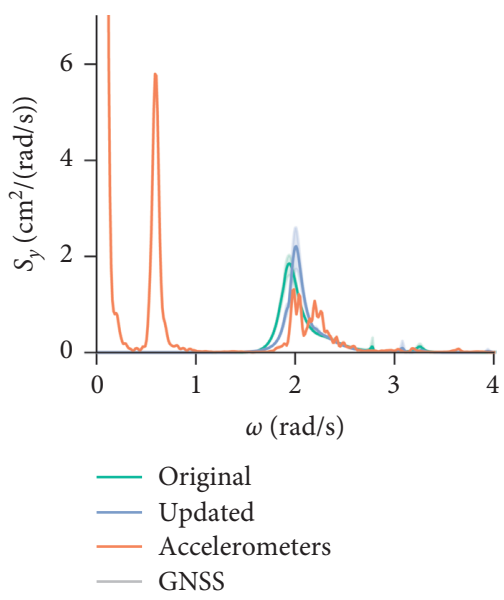

(a)

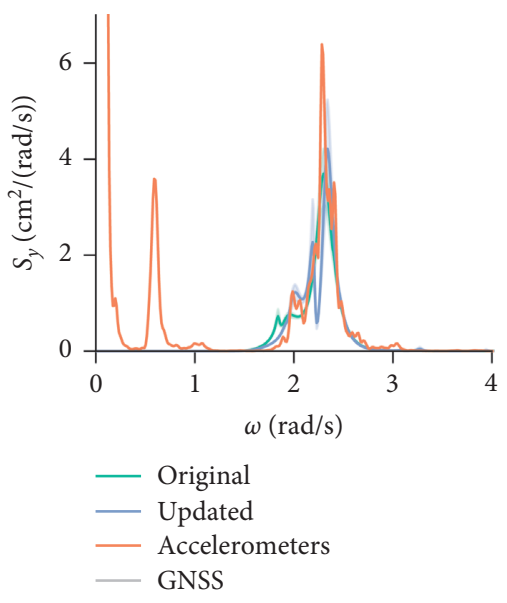

(b)

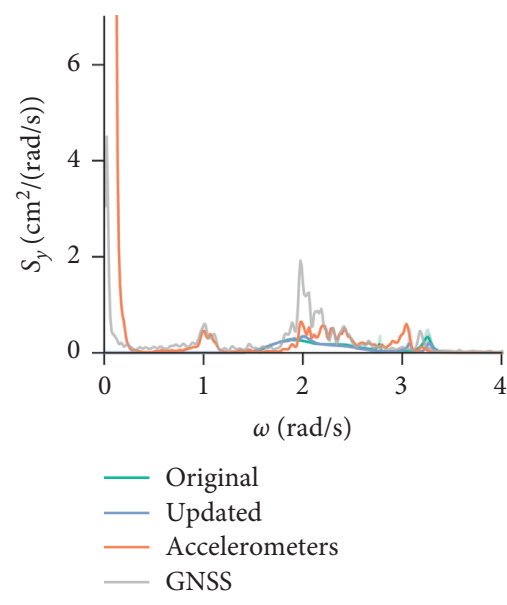

(c)

Figure 19: Comparison between the measured and predicted lateral displacement spectral densities from the selected recording, corresponding to the wave spectral density presented in Figure 18. Original and Updated refer to the original and updated numerical models, respectively. (a) Pontoon 2. (b) Pontoon 3. (c) Pontoon 4.

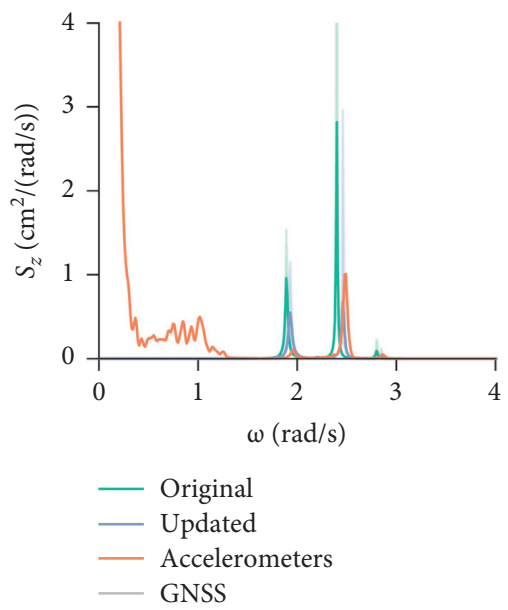

(a)

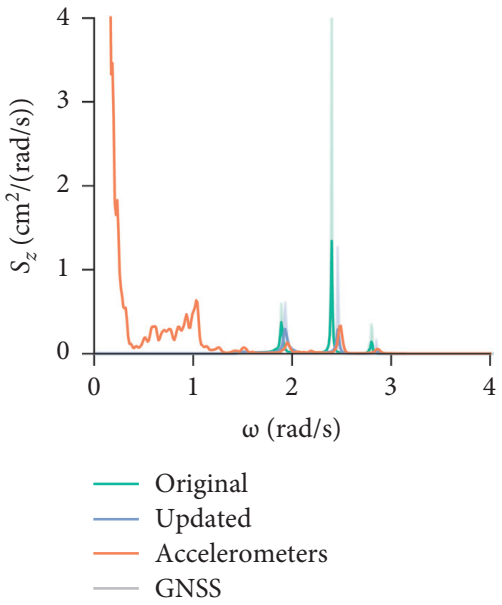

(b)

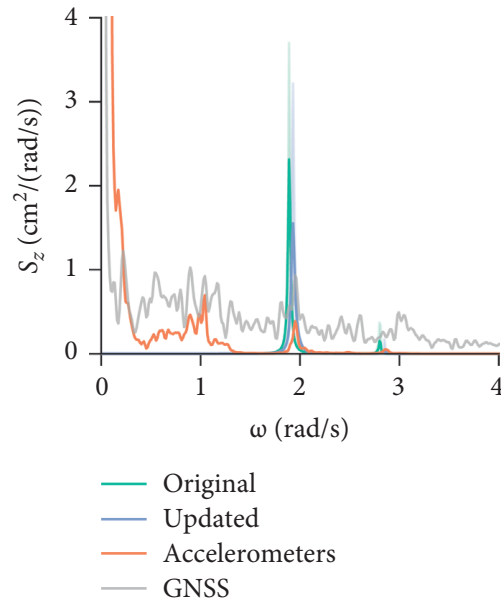

(c)

Figure 20: Comparison between the measured and predicted vertical displacement spectral densities from the selected recording, corresponding to the wave spectral density presented in Figure 18. Original and Updated refer to the original and updated numerical models, respectively. (a) Pontoon 2. (b) Pontoon 3. (c) Pontoon 4.

responding modes do not have large hydrodynamic damping contributions, their total damping are to a large extent controlled by $\xi_{0}$. To illustrate the effect the uncertain damping has on the response, shaded regions are included in the figures, to represent the response spectral densities predicted with $\xi_{0} \in[0.2 \%, 1.0 \%]$. The plots support that the amplitude of the peaks corresponding to many of the modes is highly dependent on the choice of the damping of the dry part of the structure, characterized by $\xi_{0}$.

The predicted and measured standard deviations of lateral, vertical, and torsional acceleration of all pontoons corresponding to the sea state in the chosen recording are shown in Figure 22. A similar observation as with the spectral density comparison can be made; the updated model improves both the already quite good agreement of the lateral response and significantly improves the agreement of the torsional response. However, the agreement between measured and predicted vertical response is apparently worsened by the model update by investigation of this figure. As shown in Figure 20, this is not the case. The discrepancies are larger for all peaks present in the model predictions using the original model compared to the updated model; however, the larger values do to some extent compensate for the missing frequency content around $1 \mathrm{rad} /$ $s$ present in both predictions, falsely giving the impression that the model update worsens the accuracy. The plot also 

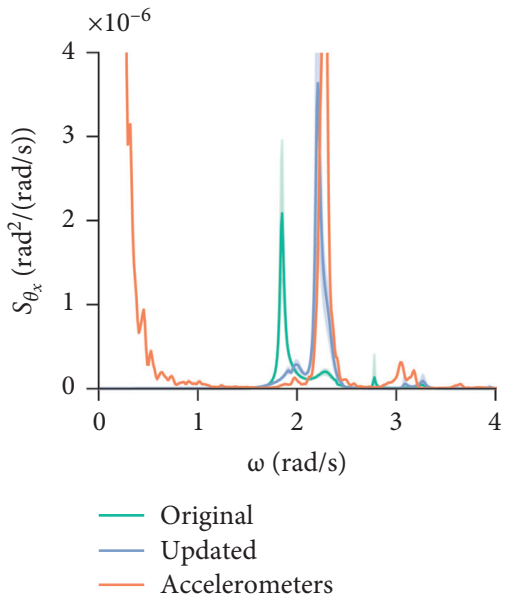

(a)
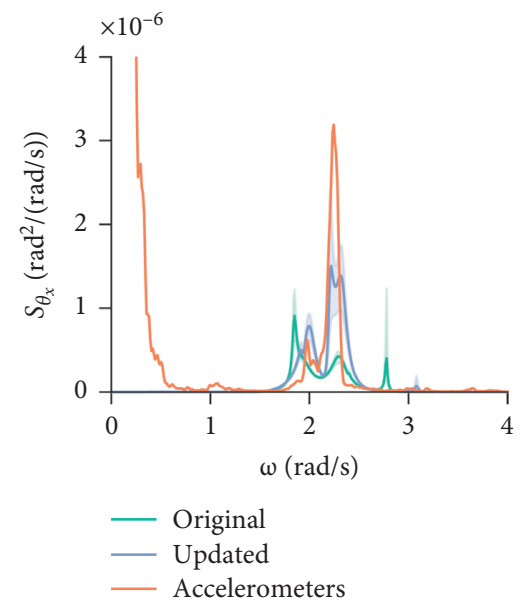

(b)
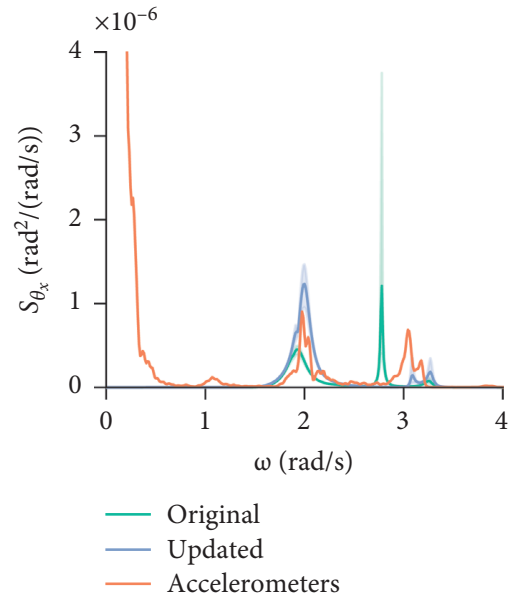

(c)

Figure 21: Comparison between the measured and predicted torsional displacement spectral densities from the selected recording, corresponding to the wave spectral density presented in Figure 18. Original and Updated refer to the original and updated numerical models, respectively. (a) Pontoon 2. (b) Pontoon 3. (c) Pontoon 4.

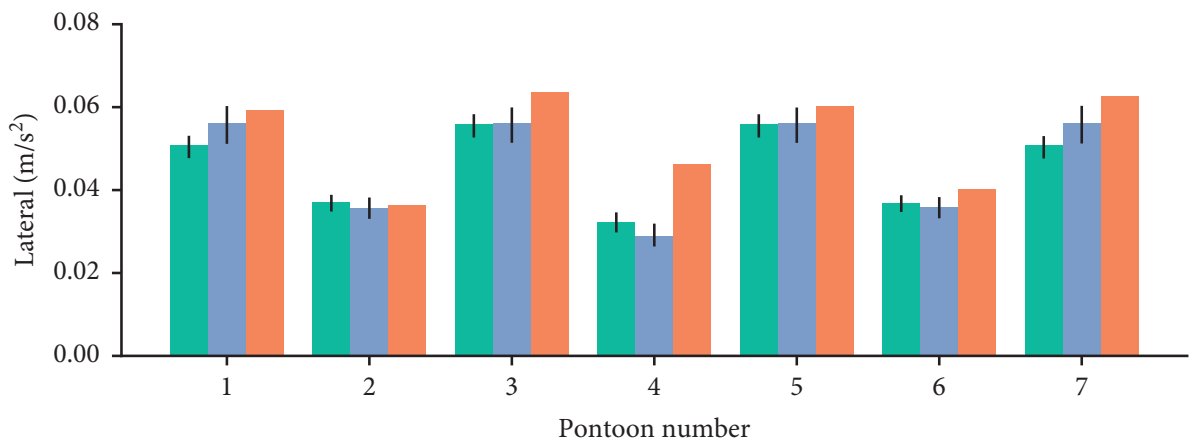

Prediction (original)
Prediction (updated)

Measurement

(a)

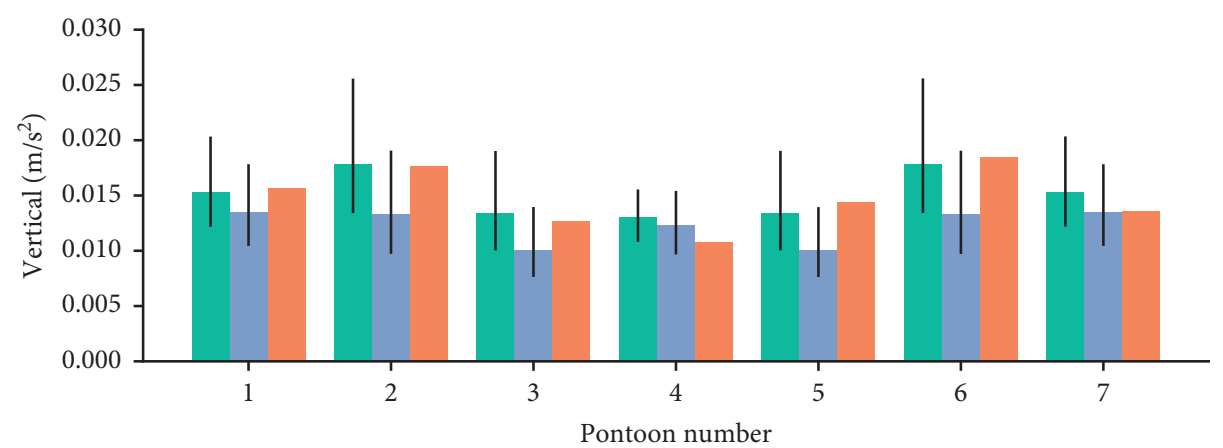

Prediction (original)

Prediction (updated)

Measurement

(b)

Figure 22: Continued. 


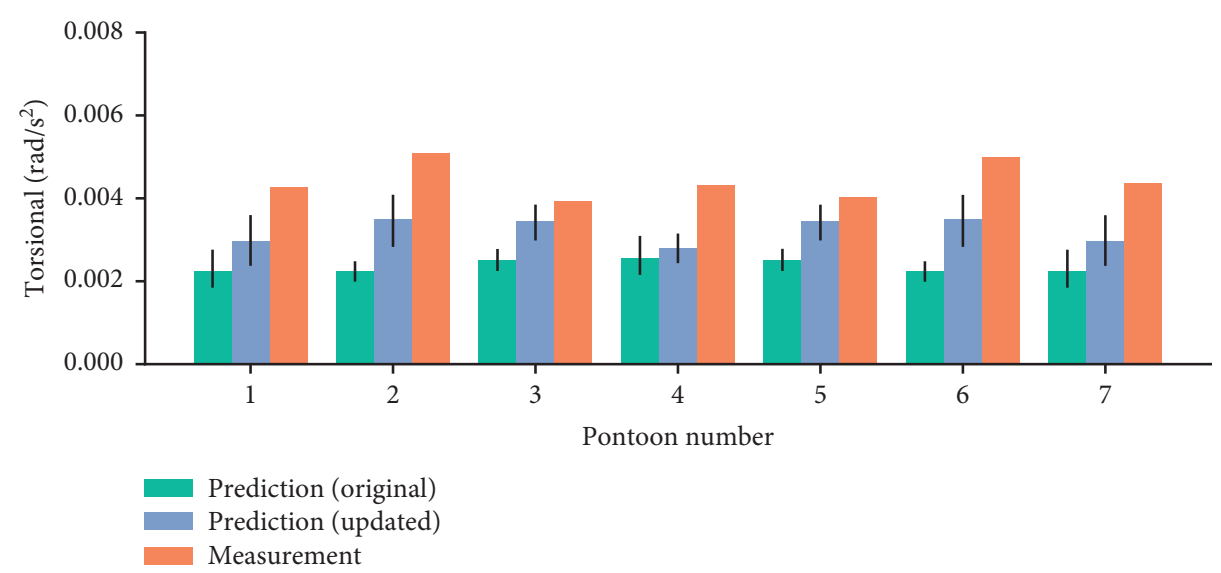

(c)

FIGURE 22: Standard deviations of predicted (original and updated models) and measured accelerations from wave excitation given by the sea state from the selected recording. The black error bars indicate the resulting response ranges when varying the modal dry critical damping ratio as $\xi_{0} \in[0.2 \%, 1.0 \%]$.

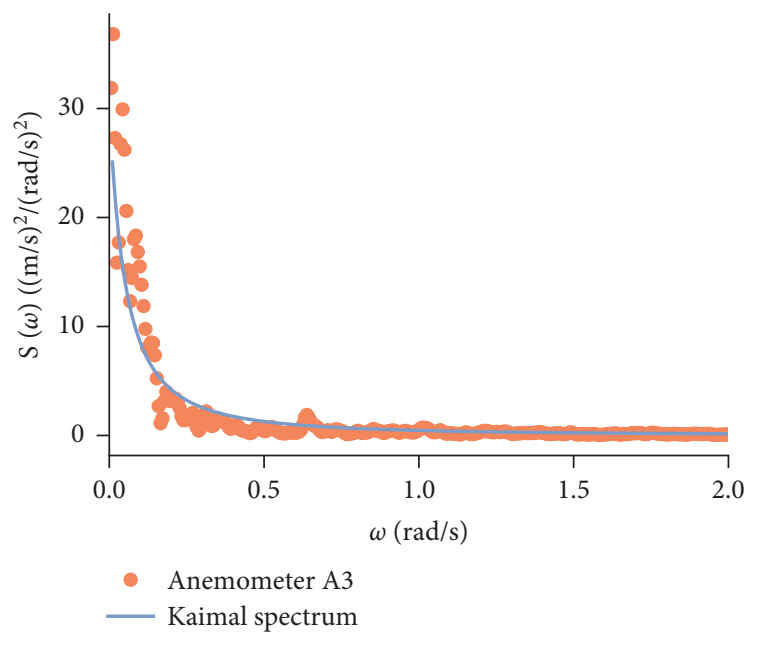

(a)

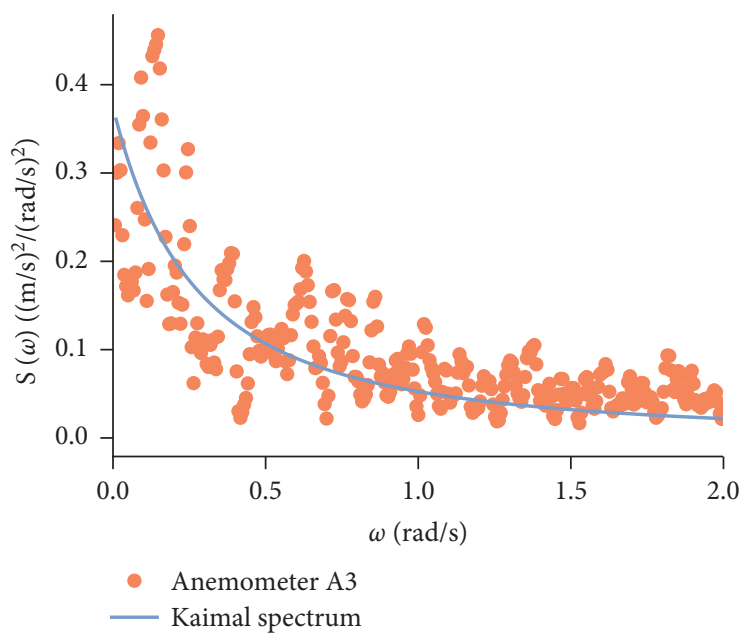

(b)

Figure 23: Wind turbulence spectral density estimates from anemometer A3 and fitted Kaimal spectra. (a) Horizontal wind. (b) Vertical wind.

shows error bars to assess the effect of the uncertainty of the modal structural damping. The given sea state causes vertical response of modes with a low hydrodynamic damping contribution and, thus, the damping ratio has a very large effect on the vertical response.

4.2.1. Approximating the Effect of Wind. The first (lateral) mode of the bridge, located around $0.6 \mathrm{rad} / \mathrm{s}$, is contributing to a significant lateral response that is not captured by the numerical predictions. Furthermore, a wide-banded vertical response around $1 \mathrm{rad} / \mathrm{s}$ is not captured by the prediction model. This is consistent with a wave excitation without energy in that frequency range, as seen in Figure 18; it is, therefore, likely that the response observed in the measurements is caused by wind excitation. To assess the effect wind excitation could have on the response, an approximate quasi-steady formulation was included in the model setup. The specifics of the modelling of the aerodynamic buffeting and self-excited forces are given in the appendix.

The following static wind coefficients were applied herein: drag coefficient $C_{d}=0.74$, lift coefficient $C_{l}=1.01$, and pitch moment coefficient $C_{m}=0.16$, which are taken from a wind engineering report related to the design of the bridge [26]. As described in Table 2, the recording is characterized by winds with a mean direction of $105^{\circ}$ (origin, positive clockwise) and mean wind speeds $U$ around $17 \mathrm{~m} / \mathrm{s}$. This is assumed as the conditions of the wind over the full length of the bridge. It is furthermore assumed that the wind characteristics do not vary over the altitude. The standard deviations of the wind amplitudes of the recording are $\sigma_{u}=2 \mathrm{~m} / \mathrm{s}$ (horizontal wind) and $\sigma_{w}=$ $0.5 \mathrm{~m} / \mathrm{s}$ (vertical wind). 


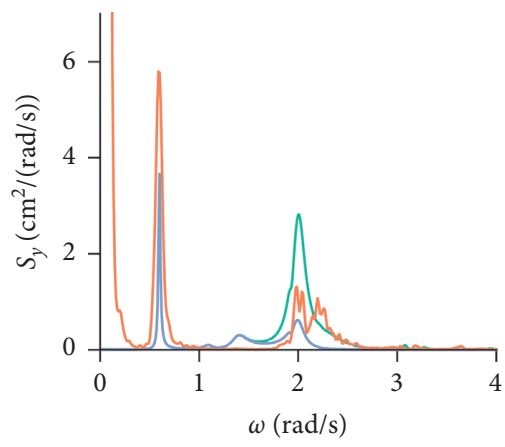

Wind and waves
Wind
Accelerometers

(a)

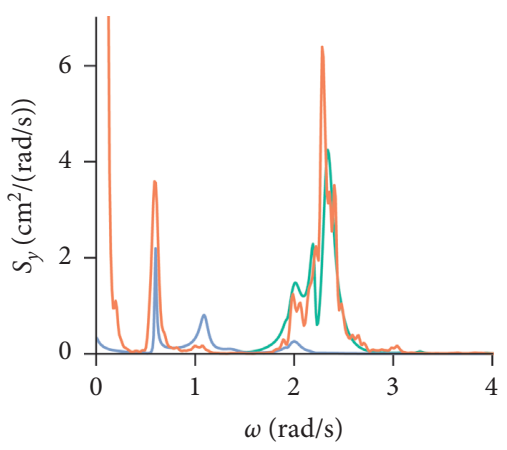

Wind and waves
Wind
Accelerometers

(b)

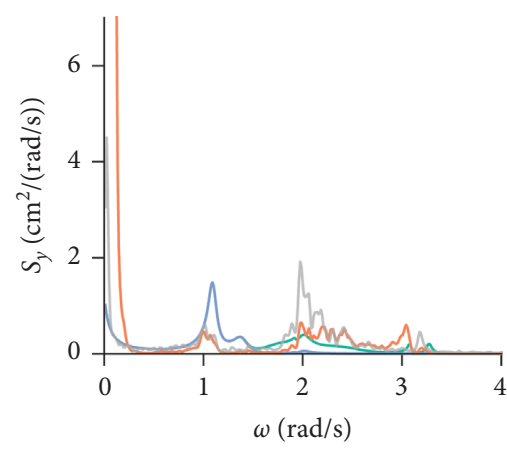

W Wind and waves

- Wind

_ Accelerometers

- GNSS

(c)

FIGURE 24: Comparison between the measured and predicted lateral displacement spectral densities from the selected recording, including approximate wind contribution. (a) Pontoon 2. (b) Pontoon 3. (c) Pontoon 4.

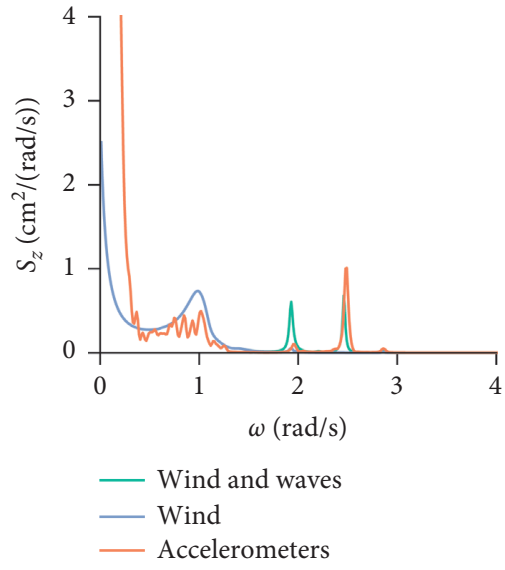

(a)

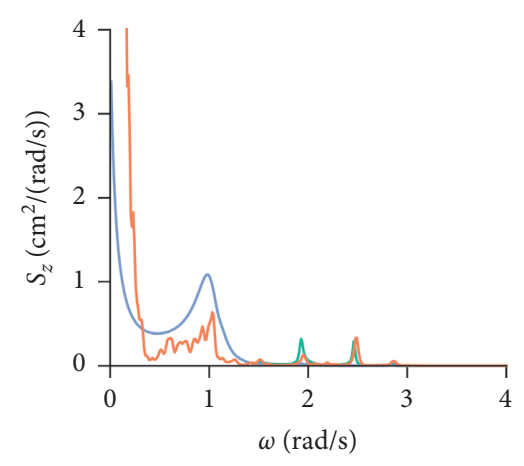

— Wind and waves
— Wind
- Accelerometers

(b)
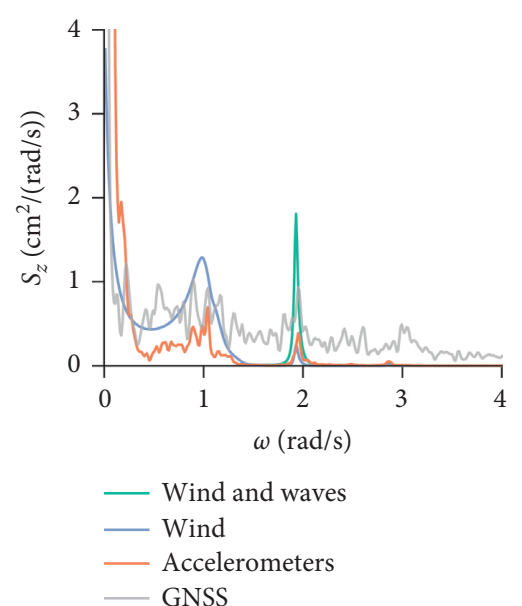

(c)

Figure 25: Comparison between the measured and predicted vertical displacement spectral densities from the selected recording, including approximate wind contribution. (a) Pontoon 2. (b) Pontoon 3. (c) Pontoon 4.

The turbulence spectral density estimated based on the wind measured by anemometer A3 is thereafter manually fitted to a Kaimal-type spectrum [27] (Figure 23), which reads as follows for component $i=u, w$ :

$$
\begin{aligned}
S_{i}(\omega) & =\frac{A_{i} f_{z}}{\left(1+1.5 A_{i} f_{z}\right)^{5 / 3}} \cdot \sigma_{i}^{2} \cdot \frac{2 \pi}{\omega}, \\
f_{z} & =\frac{\omega}{2 \pi} \frac{z}{U} .
\end{aligned}
$$

Furthermore, the coherence of the wind turbulence is specified by Davenport's expression [28], which for component $i$ between positions with a distance $\Delta x$ along the main span direction, reads as follows:

$$
\gamma(f, \Delta x)=\exp \left(-C_{i} \frac{f \Delta x}{U}\right),
$$

where $C_{i}$ is the decay coefficient for component $i$. For the current analysis, the following parameters were assumed: $B=11 \mathrm{~m}, D=7 \mathrm{~m}, A_{u}=200$, and $A_{w}=40$.

Figures 24 and 25 show the response of the lateral and vertical components, respectively, predicted with the updated model, when the estimated buffeting action and self-excited forces are both included. A significant improvement of the lower frequency ranges is observed, yielding a good agreement for most components. It is reasonable that the remaining discrepancies observed in the low-frequency domain are due to inaccuracies in the wind modelling. However, distortion of the response of the lowest 
frequencies in the accelerometers would also, together with the factor $\omega^{-4}$ to transform to displacement spectral densities, render the measurement-based displacement amplitudes of the response in the low-frequency regime highly uncertain.

\section{Concluding Remarks}

The wave-induced response of an existing floating pontoon bridge was predicted and compared with the corresponding measured acceleration response. For each recording, a JONSWAP spectrum was fitted to the average of the spectral density estimates from all wave radars. A decent agreement was observed for the lateral and vertical responses, whereas the torsional acceleration was found to be significantly underpredicted. When predicting the response for all recordings with a wave spectral density estimate reasonably close to a JONSWAP spectrum, the overall fit of the predictions was assessed for each DOF; this analysis supported the above-mentioned findings. By fitting linear functions to describe the $H_{s}$ relation to $T_{p}$ and $\gamma$, $H_{s}$ was used as the only characterizing excitation parameter. This approach gave response predictions that for most DOFs were in agreement with the predictions performed with all parameters retained.

A chosen recorded sea state was furthermore used for a more in-depth investigation of the predicted and measured response. By utilizing the numerical model obtained after a sensitivity-based model updating technique, the predictions' agreement to the measured response improved a lot, particularly for vertical and torsional components. The response of the modes below the frequency region of the wave excitation (below $1.5 \mathrm{rad} / \mathrm{s}$ ) suggests that wind action contributes significantly to the response. By applying a simplified quasi-steady wind formulation, the response induced by winds was approximated. The resulting predicted displacement response, induced by both wave and wind excitation, agree reasonably well with the measured response for most components. Measurement error in the very lowfrequency regions of the acceleration measurements are exaggerated by the transformation to displacements, leading to a large uncertainty for low-frequency components. Furthermore, a more refined formulation of the wind action would likely improve the quality of the predictions.

The response effects of the inherent uncertainty of the assumed structural damping were studied by varying the value of the critical damping ratio within a realistic range $(0.2 \%-1.0 \%)$. The vertical response is highly affected by this uncertainty for the studied sea state, as the hydrodynamic damping contributions are not significant for the modes excited; an increase of $90 \%$ of the vertical response is observed for several pontoons for critical damping ratios in the given range.

For most engineering purposes, the prediction accuracy observed using the original model will likely be acceptable. The model update increases the prediction accuracy significantly, indicating that accurately modelling structures of this kind might be challenging. A thorough screening of potential critical combinations of the environmental parameters, affecting the excitation frequency range, is therefore very important in the design process. Despite being quite successful in reproducing the measured response, the study emphasizes that floating bridges are complex structures prone to dynamic behaviour. Consequently, they are not always behaving as expected. Also, measurement campaigns of existing structures, verification studies of numerical models, and surveys to enable a better description of the environmental conditions prior to design phases are all considered crucial aspects when pursuing longer spans for floating bridges.

\section{Appendix}

The aerodynamic self-excited forces can be expressed in terms of aerodynamic stiffness and damping contributions per unit length, which traditionally are represented by aerodynamic derivatives (ADs), as proposed in [29]:

$$
\begin{gathered}
{\left[k_{\mathrm{ae}}(\omega)\right]=\frac{\rho B^{2}}{2} \omega^{2}\left[\begin{array}{ccc}
P_{4}^{*} & P_{6}^{*} & B P_{3}^{*} \\
H_{6}^{*} & H_{4}^{*} & B H_{3}^{*} \\
B A_{6}^{*} & B A_{4}^{*} & B^{2} A_{3}^{*}
\end{array}\right],} \\
{\left[c_{\mathrm{ae}}(\omega)\right]=\frac{\rho B^{2}}{2} \omega\left[\begin{array}{ccc}
P_{1}^{*} & P_{5}^{*} & B P_{2}^{*} \\
H_{5}^{*} & H_{1}^{*} & B H_{2}^{*} \\
B A_{5}^{*} & B A_{1}^{*} & B^{2} A_{2}^{*}
\end{array}\right] .}
\end{gathered}
$$

Here, $D$ is the height of the cross section, $B$ is the depth of the cross section, $\rho$ is the air density, $P_{i}^{*}, H_{i}^{*}$, and and $A_{i}^{*}$ are the dimensionless Ads, and the matrices are expressing the lateral, vertical, and rotational components of the selfexcited forces. By using quasi-steady theory, the dimensionless ADs can be approximated as follows:

$$
\begin{aligned}
& P_{1}^{*}=-2 \bar{C}_{d} \frac{D}{B} \frac{1}{K}, \\
& P_{5}^{*}=\left(\bar{C}_{l}-C_{d^{\prime}} \frac{D}{B}\right) \frac{1}{K}, \\
& P_{3}^{*}=C_{d^{\prime}} \frac{D}{B} \frac{1}{K^{2}}, \\
& H_{5}^{*}=-2 \bar{C}_{l} \frac{1}{K}, \\
& A_{5}^{*}=-2 \bar{C}_{m} \frac{1}{K}, \\
& P_{2}^{*}=P_{4}^{*}=P_{6}^{*}=H_{6}^{*}=A_{6}^{*}=0,
\end{aligned}
$$

where $K$ is the reduced frequency, defined as $K=B \omega / U, U$ is the mean wind speed, and $\bar{C}_{d, l, m}$ and $C_{d, l, m}^{\prime}$ are the values of the linearized drag, lift, and moment coefficients, 
respectively, defining their values at zero and tangent at zero. The frequency-domain transfer function matrix relating wind turbulence to buffeting force is given as follows if admittance terms are neglected:

$$
\left[B_{q}(\omega)\right]=\frac{\rho U B}{2}\left[\begin{array}{cc}
2 \frac{D}{B} \bar{C}_{d} & \frac{D}{B} C_{d}^{\prime}-\bar{C}_{l} \\
2 \bar{C}_{l} & C_{l}^{\prime}+\frac{D}{B} \bar{C}_{d} \\
2 B \bar{C}_{m} & B C_{m}^{\prime}
\end{array}\right] .
$$

The buffeting action per unit length can finally be written as follows in frequency domain:

$$
\left\{G_{\mathrm{ae}}(\omega)\right\}=\left[B_{q}(\omega)\right]\left\{G_{\text {turb }}(\omega)\right\},
$$

where $\left\{G_{\text {turb }}(\omega)\right\}$ is the frequency-domain representation of the turbulent component of the wind, most commonly represented by spectral densities. The aerodynamic stiffness, the aerodynamic damping, and the buffeting forces can either be integrated over the full bridge length using the mode shapes, or over each element in a finite element approach as in [30], to yield the total contributions. The aerodynamic contributions can be combined with the structural and hydrodynamic contributions as follows:

$$
\begin{aligned}
& \left(\left[M_{s}\right]+\left[M_{h}(\omega)\right]\right)\{\ddot{u}\}+\left(\left[C_{s}\right]+\left[C_{h}(\omega)\right]-\left[C_{a e}(\omega)\right]\right)\{\dot{u}\} \\
& \quad+\left(\left[K_{s}\right]+\left[K_{h}\right]-\left[K_{\mathrm{ae}}(\omega)\right]\right)\{u\}=\left\{P((\omega)\} e^{i \omega t} .\right.
\end{aligned}
$$

\section{Data Availability}

The data used to support the findings of the study are available from the corresponding author upon request.

\section{Conflicts of Interest}

The authors declare that they have no conflicts of interest.

\section{Acknowledgments}

This research was conducted with financial support from the Norwegian Public Roads Administration. The authors gratefully acknowledge this support.

\section{References}

[1] I. Langen and R. Sigbjörnsson, "On stochastic dynamics of floating bridges," Engineering Structures, vol. 2, no. 4, pp. 209-216, 1980.

[2] K. A. Kvåle, R. Sigbjörnsson, and O. Øiseth, "Modelling the stochastic dynamic behaviour of a pontoon bridge: a case study," Computers \& Structures, vol. 165, pp. 123-135, 2016.

[3] M. S. Seif and Y. Inoue, "Dynamic analysis of floating bridges," Marine Structures, vol. 11, no. 1-2, pp. 29-46, 1998.
[4] Ø. W. Petersen and O. Øiseth, "Sensitivity-based finite element model updating of a pontoon bridge," Engineering Structures, vol. 150, pp. 573-584, 2017.

[5] F.-I. G. Giske, K. A. Kvåle, B. J. Leira, and O. Øiseth, "Longterm extreme response analysis of a long-span pontoon bridge," Marine Structures, vol. 58, pp. 154-171, 2018.

[6] B. J. Hartz, "Dynamic response of the Hood canal floating bridge," in Proceedings Second ASCE/EMD Specialty Conference on Dynamic Response of Structures, Atlanta, GA, USA, January 1981.

[7] E. R. Morris, "A dynamic analysis of the okanagan lake floating bridge," Master thesis, University of British Columbia, Vancouver, Canada, 1999.

[8] N. Kumamoto and T. Maruyama, "Elastic response analysis method for floating bridges in waves," Technical report, Mitsubishi Heavy Industries, Ltd., Tokyo, Japan, 2000.

[9] E. Watanabe, T. Maruyama, H. Tanaka, and S. Takeda, "Design and construction of a floating swing bridge in Osaka," Marine Structures, vol. 13, no. 4-5, pp. 437-458, 2000.

[10] Z. Cheng, Z. Gao, and T. Moan, "Wave load effect analysis of a floating bridge in a fjord considering inhomogeneous wave conditions," Engineering Structures, vol. 163, pp. 197-214, 2018.

[11] Y. Xu, O. Øiseth, and T. Moan, "Time domain simulations of wind- and wave-induced load effects on a three-span suspension bridge with two floating pylons," Marine Structures, vol. 58, pp. 434-452, 2018.

[12] T. Viuff, B. J. Leira, X. Xiang, and O. Øiseth, "Effects of wave directionality on extreme response for a long end-anchored floating bridge," Applied Ocean Research, vol. 90, p. 101843, 2019.

[13] T. Viuff, X. Xiang, O. Øiseth, and B. J. Leira, "Model uncertainty assessment for wave- and current-induced global response of a curved floating pontoon bridge," Applied Ocean Research, vol. 105, p. 102368, 2020.

[14] X. Xiang and A. Løken, Hydroelastic Analysis and Validation of an End-Anchored Floating Bridge Under Wave and Current Loads, American Society of Mechanical Engineers, New York, NY, USA, 2019.

[15] C. Georgiadis, "Wave induced vibrations of continuous floating structures," Doctoral dissertation, University of Washington, Seattle, WA, USA, 1981.

[16] S. T. Peterson, "Experimental response and analysis of the evergreen point floating bridge," Doctoral dissertation, Washington State University, Seattle, WA, USA, 2002.

[17] K. A. Kvåle and O. Øiseth, "Structural monitoring of an endsupported pontoon bridge," Marine Structures, vol. 52, pp. 188-207, 2017.

[18] K. A. Kvåle and O. Øiseth, "Characterization of the wave field around an existing end-supported pontoon bridge from simulated data," in Proceedings of the International Conference on Earthquake Engineering and Structural Dynamics, R. Rupakhety, S. Olafsson, and B. Bessason, Eds., Springer International Publishing, Berlin, Germany, pp. 345-359, 2019.

[19] A. Naess and T. Moan, Stochastic Dynamics of Marine Structures, Cambridge University Press, New York, NY, USA, 2012.

[20] K. Hasselmann, T. P. Barnett, E. Bouws et al., "Measurements of wind-wave growth and swell decay during the joint north sea wave project (JONSWAP)," Ergänzungsheft zur Deutschen Hydrographischen Zeitschrift, Reihe A, no. 12., 1973.

[21] C. T. Stansberg, G. Contento, S. Won Hong et al., "The specialist committee on waves: final report and 
recommendations to the 23rd ITTC," in Proceedings of the 23rd ITTC, pp. 505-736, Venice, Italy, September 2002.

[22] M. S. Longuet-Higgins, D. E. Cartwright, and N. D. Smith, "Observations of the directional spectrum of sea waves using the motions of a floating buoy," in Proceedings of the Conference Ocean Wave Spectra, pp. 111-132, Prentice-Hall, Easton, ML, USA, May 1963.

[23] $\mathrm{P}$ Vegdirektoratet, Rose design rules for the tender phase, Bergsøysundet bridge, Møre og Romsdal county, Trollstigen, MORE, 1989.

[24] P. D. Welch, "The use of fast fourier transform for the estimation of power spectra: a method based on time averaging over short, modified Periodograms," IEEE Transactions on Audio and Electroacoustics, vol. 12, 1967.

[25] R. E. Walpole, R. H. Myers, S. L. Myers, and K. Ye, Probability and Statistics for Engineers and Scientists, Pearson, London, UK, 8 edition, 2007.

[26] T. Søreide, "Bergsøysundet bridge, Control report: Wind analysis," Technical Reort, 1991.

[27] J. C. Kaimal, J. C. Wyngaard, Y. Izumi, and O. R. Coté, "Spectral characteristics of surface-layer turbulence," Quarterly Journal of the Royal Meteorological Society, vol. 98, no. 417 , pp. 563-589, 1972.

[28] A. G. Davenport, "The spectrum of horizontal gustiness near the ground in high winds," Quarterly Journal of the Royal Meteorological Society, vol. 87, no. 372, pp. 194-211, 1961.

[29] R. H. Scanlan and J. J. Tomko, "Airfoil and bridge deck flutter derivatives," Journal of the Engineering Mechanics Division, vol. 97 , no. 6, pp. 1717-1737, 1971.

[30] O. Øiseth, A. Rönnquist, and R. Sigbjörnsson, "Finite element formulation of the self-excited forces for time-domain assessment of wind-induced dynamic response and flutter stability limit of cable-supported bridges," Finite Elements in Analysis and Design, vol. 50, pp. 173-183, 2012. 